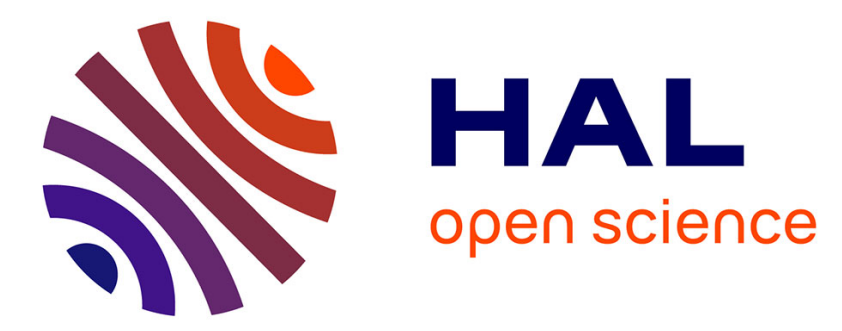

\title{
Dual-Hop Spatial Modulation With a Relay Transmitting Its Own Information
}

Qiang Li, Miaowen Wen, Marco Di Renzo, Vincent Poor, Shahid Mumtaz, Fangjiong Chen

\section{- To cite this version:}

Qiang Li, Miaowen Wen, Marco Di Renzo, Vincent Poor, Shahid Mumtaz, et al.. Dual-Hop Spatial Modulation With a Relay Transmitting Its Own Information. IEEE Transactions on Wireless Communications, 2020, 19 (7), pp.4449 - 4463. 10.1109/TWC.2020.2983696 . hal-03020403

\section{HAL Id: hal-03020403 https://hal.science/hal-03020403}

Submitted on 24 Nov 2020

HAL is a multi-disciplinary open access archive for the deposit and dissemination of scientific research documents, whether they are published or not. The documents may come from teaching and research institutions in France or abroad, or from public or private research centers.
L'archive ouverte pluridisciplinaire HAL, est destinée au dépôt et à la diffusion de documents scientifiques de niveau recherche, publiés ou non, émanant des établissements d'enseignement et de recherche français ou étrangers, des laboratoires publics ou privés. 


\title{
Dual-Hop Spatial Modulation With A Relay
}

\section{Transmitting Its Own Information}

\author{
Qiang $\mathrm{Li}^{\dagger}$, Miaowen $\mathrm{Wen}^{\dagger}$, Senior Member, IEEE, \\ Marco Di Renzo ${ }^{\#}$, Fellow, IEEE, H. Vincent Poor $^{\$}$, Life Fellow, IEEE, \\ Shahid Mumtaz ${ }^{\star}$, Senior Member, IEEE, and Fangjiong Chen ${ }^{\dagger}$, Member, IEEE \\ ${ }^{\dagger}$ School of Electronic and Information Engineering, \\ South China University of Technology, Guangzhou 510640, China \\ \#Université Paris-Saclay, CNRS, CentraleSupélec, \\ Laboratoire des Signaux et Systèmes, 91192 Gif-sur-Yvette, France \\ ${ }^{\$}$ Department of Electrical Engineering, Princeton University, Princeton, NJ 08544, USA \\ ${ }^{\star}$ Instituto de Telecomunicações, Aveiro 3810-193, Portugal \\ Email: eeqiangli@mail.scut.edu.cn, eemwwen@scut.edu.cn, \\ marco.direnzo@centralesupelec.fr, poor@princeton.edu, \\ smumtaz@av.it.pt, eefjchen@scut.edu.cn
}

\begin{abstract}
In this paper, a novel dual-hop spatial modulation (SM) relay network is proposed, in which the relay is enabled to transmit its own information while forwarding the SM signal from the source over the same frequency band. Both decode-and-forward (DF) and amplify-and-forward (AF) relaying protocols are studied. For DF relaying, the relay embeds its own information into one out of two spatial dimensions of quadrature SM. For AF relaying, the relay activates only one transmit antenna to forward the received signal, and encodes its own information by the index of the active antenna. Under both relaying protocols, the throughput of the network is increased without consuming extra power. The bit error rate (BER) performance is analyzed, and tight upper bounds on the BERs are derived in closed form for the source and relay over Rayleigh fading channels for both DF and AF relaying. The performance analysis is verified by Monte Carlo simulations, showing that the proposed scheme provides a simple yet effective solution to the implementation of SM relay networks in which the relay has its own information to be transmitted.
\end{abstract}

\section{Index Terms}

Amplify-and-forward (AF), bit error rate, decode-and-forward (DF), performance analysis, quadrature spatial modulation. 


\section{INTRODUCTION}

Spatial modulation (SM) is a promising digital modulation technology that uses the transmit antennas of a multiple-input multiple-output (MIMO) system in an innovative fashion [1]-[7]. In SM, only a single transmit antenna is activated for each transmission of a constellation symbol. Specifically, extra information bits, termed spatial bits, are conveyed through the index of the active antenna. The active antenna is dynamically altered according to the spatial bits to be transmitted. Only one radio-frequency chain is required at the SM transmitter and the interchannel interference is completely avoided at the receiver. SM achieves an appealing trade-off between the spectral efficiency and energy efficiency with a low implementation cost. Based on the SM philosophy, numerous variants of SM technology have emerged, which typically include generalized SM [8], [9], receive SM (RSM) [10], differential SM [11], [12], space shift keying (SSK) [13], [14], and quadrature SM (QSM) [15]. In particular, by applying the concept of SM at the receiver side, RSM uses the indices of receive antennas to convey spatial bits in addition to conventional constellation symbols. SSK solely adopts the index of an active antenna to carry information, enjoying a concise transceiver architecture at the expense of spectral efficiency. QSM introduces another spatial dimension for information embedding. In QSM, the real and imaginary parts of a constellation symbol may be transmitted from two different antennas, whose indices are determined by two different spatial bit streams. Consequently, QSM doubles the number of spatial bits and maintains almost all inherit advantages of SM with the same number of transmit antennas.

Recently, SM has also been studied in non-cooperative/cooperative relay networks. Relaying is an effective way to enhance the network reliability and enlarge the network coverage [16], [17]. Different relaying protocols that strike different trade-offs between performance and complexity, such as amplify-and-forward (AF) and decode-and-forward (DF) relaying, can be employed at the relay. In [18]-[22], the SM technology is amalgamated with half-duplex DF relaying. More specifically, in [18], SM is employed at the source in a dual-hop DF relaying for the sake of transmitting additional bits. A cooperative DF relay-assisted SM scheme is proposed in [19] by considering the different importance of spatial bits and constellation bits in SM detection. In [20], an SM transmitter cooperates with multiple DF relays, and only the relays that correctly detect the received signal forward the decoded data to the destination. The study of [21] conceives a 
virtual MIMO non-cooperative DF relay network, where RSM and SM are invoked in the first and second hops, respectively. Inspired by the essence of SM, [22] develops a cooperative SM-aided non-orthogonal multiple access system, in which the messages for two users are conveyed by two different information-bearing units of SM. For SM-based AF relaying systems, [23] presents a cooperative SM system with a single AF relay, while [24] studies the performance of QSM with multiple AF relays. Unlike the studies in [18]-[24] where half-duplex relays are assumed, [25]-[27] investigate the potential of SM in full-duplex relaying by exploiting the inherent nature of SM that only one transmit antenna is activated per transmission. Particularly, in [25] and [26], the relay forwards the previously decoded information to the destination using SM, while the remaining "inactive" antennas at the relay are used for reception. In contrast to [25] and [26] where SM is applied at the DF relay, [27] proposes a dual-hop two-way full-duplex SM system, where SM is used at the sources and energy harvesting is employed at the AF relay. It is worth noting that in all above mentioned SM-based relay networks, the relays only retransmit the information from the sources, and they do not pursue any personal interests. When the relays have their own information to be transmitted, it is inevitable to occupy extra resource such as time slot and frequency band, which degrades the system throughput and increases the network latency. In [28] and [29], the two information-bearing units of SM are exploited in cognitive radio by two different sources, such that the information from the primary and secondary transmitters can be transmitted at the same time. The study of [30] introduces the scheme of distributed SM (DSM) with the aid of multiple single-antenna DF relays, in which the data from the source is encoded into the index of one active relay and the signal domain is exploited to convey the data of the relay. In DSM, the source employs $M$-phase shift keying (PSK)/quadrature amplitude modulation (QAM), and SM is performed at the relays in a distributed manner. More recently, a similar idea of using a reconfigurable intelligent surface (RIS) for reflecting the imping signals and carrying RIS data via the indices of the RIS elements is proposed in [31].

To the best of our knowledge, however, in an SM relay network where SM is invoked at the source, how to convey private information of the relay while forwarding the message from the source to the destination, is still an open issue. To fill this gap, in this paper, we propose a novel dual-hop SM relay network with relay's private information, termed SMRN-RPI, where both DF and AF relaying protocols are developed for the relay. SMRN-RPI has potential applications in various relay-aided communications in which both the source and relay have information to be 
transmitted. One typical example is the local program insertion in digital video broadcasting-next generation handheld [32]. The contributions of this paper are summarized as follows.

- A novel dual-hop MIMO architecture based on SM/QSM is established, in which SM is employed at a source, and a DF/AF relay forwards the message from the source and concurrently transmits its private information to a destination using QSM/SM. More specifically, for DF relaying, after decoding the SM signal from the source, the relay encodes the decoded data and its private information into a QSM signal, of which one out of the two spatial dimensions, namely the in-phase (I-) and quadrature (Q-) branches, is used to convey the private information; for AF relaying, the relay amplifies and forwards the received signal to the destination through one active transmit antenna, whose index carries the information of the relay.

- For both DF and AF relaying, we analyze the average bit error probability (ABEP) performance of SMRN-RPI over Rayleigh fading channels assuming the optimal/simplified maximum-likelihood (ML) detection. Tight upper bounds on the ABEPs are derived in closed form for the source and the relay. From the theoretical expressions, the effects of different system parameters on the ABEP performance are revealed.

- Extensive Monte Carlo simulations are performed to evaluate the error performance and validate the performance analysis of SMRN-RPI with different system parameters. SMRNRPI is proved to provide a simple yet effective solution to the implementation of SM relay networks in which the relay has its own information to be transmitted.

The remainder of this paper is organized as follows. Section II describes the system model of SMRN-RPI. The performance of SMRN-RPI with DF and AF relaying is analyzed in Sections III and IV, respectively. Section V presents computer simulation results and finally Section VI concludes the paper.

Notation: Column vectors and matrices are denoted by lowercase and uppercase boldface letters, respectively. Superscripts ${ }^{T}$ and ${ }^{H}$ stand for transpose and Hermitian transpose, respectively. $h_{i}$ denotes the $i$-th entry of $\mathbf{h}, \mathbf{g}_{i}$ represents the $i$-th column of $\mathbf{G}$, and $g_{p q}$ stands for the $(p, q)$-th element of $\mathbf{G}$. The $n \times n$ identity matrix is symbolized by $\mathbf{I}_{n} \cdot \operatorname{diag}\{\cdot\}$ transforms a vector into a diagonal matrix. $\Re\{\cdot\}$ and $\Im\{\cdot\}$ return the real and imaginary parts of a complex number, respectively. $j$ is the imaginary unit. !! is the double factorial notation. $\mathcal{C N}\left(\mu, \sigma^{2}\right)$ 
TABLE I

Notations and Definitions of Some Probabilities Involved in the Performance Analysis.

\begin{tabular}{|c||c|}
\hline Notation & Definition \\
\hline \hline$P_{b R}$ & ABEP of the relay for DF/AF relaying \\
\hline$P_{I R}$ & Error probability of detecting the active antenna index (on the Q- branch) of the relay for AF (DF) relaying \\
\hline$P_{b S}$ & ABEP of the source for DF/AF relaying \\
\hline$P_{1 S}$ & ABEP of the source contributed by the symbol bits for DF/AF relaying \\
\hline$P_{1 S-S R}$ & ABEP of the source contributed by the symbol bits in the second hop for DF relaying \\
\hline$P_{1 S-R D}$ & ABEP of the source contributed by the spatial bits for DF/AF relaying \\
\hline$P_{2 S}$ & Error probability of detecting the active antenna index in the first hop for DF relaying \\
\hline$P_{I S}$ & Error probability of detecting the active antenna index of the source at the destination for DF/AF relaying \\
\hline$P_{I_{1} S}$ & Error probability of detecting the active antenna index of the source or relay for AF relaying \\
\hline$P_{I_{2} S}$ & Error probability of detecting the active antenna index on the I- branch in the second hop for DF relaying \\
\hline$P_{l_{m}}$ & Error probability of detecting the active antenna index on the I- or Q- branch of the relay for DF relaying \\
\hline$P_{l m}$ & ABributed by the symbol bits in the first hop for DF relaying \\
\hline
\end{tabular}

represents the complex Gaussian distribution with mean $\mu$ and variance $\sigma^{2}$. The probability of an event, the cumulative distribution function (CDF), and the probability density function (PDF) of a random variable are denoted by $\operatorname{Pr}(\cdot), F(\cdot)$, and $f(\cdot)$, respectively. $E\{\cdot\}, \operatorname{Var}\{\cdot\}$, and $\mathcal{L}\{\cdot\}$ denote expectation, variance, and Laplace transform, respectively. (.) and $\|\cdot\|$ stand for the binomial coefficient and Frobenius norm, respectively. $Q(\cdot), \Gamma(\cdot)$, and $\mathrm{E}_{1}(\cdot)$ represent the Gaussian $Q$-function, gamma function, and exponential integral function, respectively. $\mathbf{H}_{\nu}(\cdot)$ is the Struve function [33, Eq. (8.55)], and $Y_{i}(\cdot)$ is the Bessel function of the second kind [33, Eq. (8.403)]. $Q_{q}^{p}(\cdot)$ denotes the associated Legendre function of the second kind [33, Eq. (8.7)], and ${ }_{p} F_{q}\left(\alpha_{1}, \ldots, \alpha_{p} ; \beta_{1}, \ldots, \beta_{q} ; z\right)$ is a generalized hypergeometric series [33, Eq. (9.14)]. Table I lists the notations and definitions of some probabilities involved in the performance analysis.

\section{SySTEM MODEL}

Fig. 1 depicts the block diagram of SMRN-RPI, where a source communicates with a destination with the aid of a half-duplex DF/AF relay. Besides forwarding the information from the source, the relay has its own information to be transmitted. We assume that there is no direct link between the source and the destination, and they can only communicate with each other via the relay. The source and destination are equipped with $N_{t S}$ transmit antennas and $N_{r D}$ 


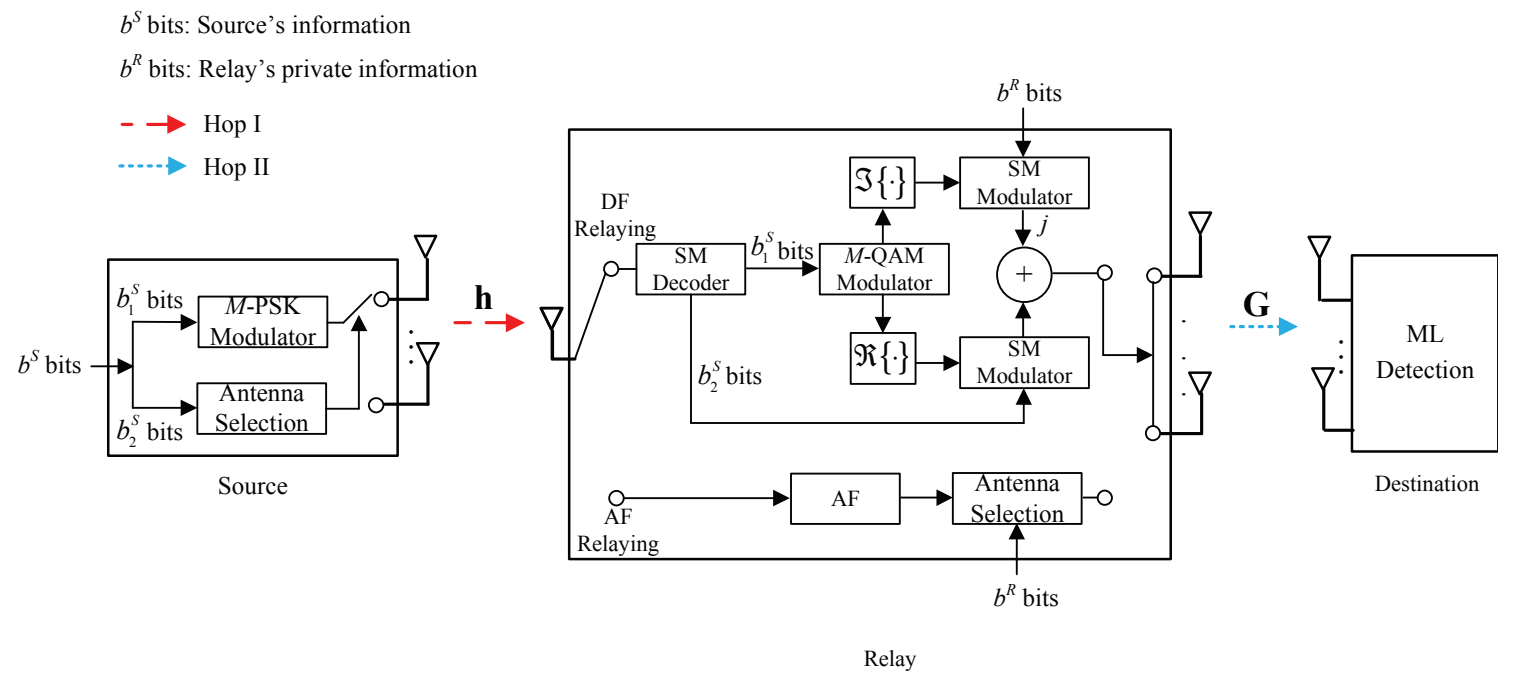

Fig. 1. Block diagram of SMRN-RPI.

receive antennas, respectively. The relay has $N_{t R}$ transmit antennas and one receive antenna. The channel matrices of the source-to-relay and relay-to-destination links are denoted by $\mathbf{h} \in \mathbb{C}^{N_{t S} \times 1}$ and $\mathbf{G} \in \mathbb{C}^{N_{r D} \times N_{t R}}$, respectively. Both channel links are assumed to experience flat Rayleigh fading. The entries of $\mathbf{h}$ and $\mathbf{G}$ are independent and identically distributed (i.i.d.) complex Gaussian random variables following distributions $\mathcal{C N}\left(0, \Omega_{S R}\right)$ and $\mathcal{C N}\left(0, \Omega_{R D}\right)$, respectively. Large scale fading is considered in this paper, and $\Omega_{S R}$ and $\Omega_{R D}$ are modeled as $\Omega_{S R}=d_{S R}^{-\alpha}$ and $\Omega_{R D}=d_{R D}^{-\alpha}$, respectively, where $\alpha$ denotes the path-loss exponent, and $d_{S R}\left(d_{R D}\right)$ is the distance of the first (second) hop [23].

For each complete transmission that consists of two hops, the source and relay convey $b^{S}$ bits and $b^{R}$ bits, respectively, to the destination. During the first hop, the source encodes $b^{S}$ bits into an SM symbol

$$
\mathbf{x}_{S}=[\underbrace{0, \ldots, 0}_{l-1}, s, \underbrace{0, \ldots, 0}_{N_{t S}-l}]^{T},
$$

and transmits it to the relay, where $s$ is a normalized $M$-ary PSK symbol determined by the $b_{1}^{S}$ bits with $|s|^{2}=1$, and $l \in\left\{1, \ldots, N_{t S}\right\}$ denotes the active antenna index determined by the $b_{2}^{S}$ bits. ${ }^{1}$ Obviously, we have $b^{S}=b_{1}^{S}+b_{2}^{S}$, where $b_{1}^{S}=\log _{2}(M)$ and $b_{2}^{S}=\log _{2}\left(N_{t S}\right)$. Here, $N_{t S}$ is

\footnotetext{
${ }^{1}$ Note that QAM signaling is also feasible. However, since PSK is more favorable than QAM for SM [34], we employ PSK signaling at the source.
} 
assumed to be an arbitrary integer power of two. Hence, $l$ can be easily obtained by converting the $b_{2}^{S}$ bits into the decimal representation. The received signal at the relay can be expressed as

$$
y_{R}=\sqrt{P_{S}} \mathbf{h}^{T} \mathbf{x}_{S}+w_{R}=\sqrt{P_{S}} h_{l} s+w_{R},
$$

where $P_{S}$ denotes the transmit power of the source, and $w_{R}$ is the additive white Gaussian noise (AWGN) at the relay, which follows the distribution $\mathcal{C N}\left(0, N_{0}\right)$. We define the transmit signalto-noise ratio (SNR) as $\rho=P_{S} / N_{0}$. During the second hop, upon reception, the relay adopts either DF or AF relaying to forward the source information and transmit its own information to the destination, which will be discussed in the next two subsections.

\section{A. DF Relaying}

Using the DF protocol, the relay first decodes the SM signal, then encodes the decoded data and relay's private information into a QSM signal, and finally conveys it to the destination. Specifically, the relay employs the optimal ML detection to jointly decode $s$ and $l$, namely

$$
(\tilde{s}, \tilde{l})=\arg \min _{s, l}\left|y_{R}-\sqrt{P_{S}} h_{l} s\right|^{2},
$$

where $\tilde{s}$ and $\tilde{l}$ are the estimates of $s$ and $l$ at the relay, respectively. The $b_{1}^{S}$ and $b_{2}^{S}$ bits are then recovered by de-mapping $\tilde{s}$ and $\tilde{l}$, respectively. The recovered $b_{1}^{S}$ bits are re-modulated into a normalized $M$-ary QAM symbol $x$. The real and imaginary parts of $x$ are further independently encoded into two SM symbols

$$
\mathbf{x}_{R}^{\Re}=[\underbrace{0, \ldots, 0,}_{\tilde{l}-1} x^{\Re}, \underbrace{0, \ldots, 0}_{N_{t R}-\tilde{l}}]^{T}, \quad \mathbf{x}_{R}^{\Im}=[\underbrace{0, \ldots, 0}_{m-1}, x^{\Im}, \underbrace{0, \ldots, 0}_{N_{t R}-m}]^{T},
$$

where $x^{\Re}=\Re\{x\}, x^{\Im}=\Im\{x\}$, and $m \in\left\{1, \ldots, N_{t R}\right\}$ is the active antenna index for the imaginary part determined by the $b^{R}$ bits of the relay. ${ }^{2}$ Obviously, we have $b^{R}=\log _{2}\left(N_{t R}\right)$ and $N_{t R} \geq N_{t S}$. After obtaining $\mathbf{x}_{R}^{\Re}$ and $\mathbf{x}_{R}^{\Im}$, the QSM symbol can be obtained as $\mathbf{x}_{R}=\mathbf{x}_{R}^{\Re}+j \mathbf{x}_{R}^{\Im}$, which is subsequently transmitted to the destination.

At the destination, the received signal can be expressed as

$$
\mathbf{y}_{D}=\sqrt{P_{R}} \mathbf{G x}_{R}+\mathbf{w}_{D}=\sqrt{P_{R}}\left(\mathbf{g}_{\tilde{l}} x^{\Re}+j \mathbf{g}_{m} x^{\Im}\right)+\mathbf{w}_{D}
$$

\footnotetext{
${ }^{2}$ When $M=2, x$ has no imaginary part, i.e., $x^{\Im}=0$. In this case, $x^{\Im}$ can simply be set to be 1 .
} 
where $P_{R}$ denotes the transmit power of the relay, and $\mathbf{w}_{D} \in \mathbb{C}^{N_{r D} \times 1}$ is the AWGN vector at the destination, which follows the distribution $\mathcal{C N}\left(\mathbf{0}, N_{0} \mathbf{I}_{N_{r D}}\right)$. According to the optimal ML detection, the information of the source and relay can be recovered from

$$
\left(\hat{l}, \hat{m}, \hat{x}^{\Re}, \hat{x}^{\Im}\right)=\arg \min _{\tilde{l}, m, x^{\Re}, x^{\Im}}\left\|\mathbf{y}_{D}-\sqrt{P_{R}}\left(\mathbf{g}_{\tilde{l}} x^{\Re}+j \mathbf{g}_{m} x^{\Im}\right)\right\|^{2},
$$

where $\hat{l}, \hat{m}, \hat{x}^{\Re}$, and $\hat{x}^{\Im}$ are the estimates of $\tilde{l}, m, x^{\Re}$, and $x^{\Im}$, respectively.

\section{B. AF Relaying}

For AF relaying, the relay first amplifies the received signal, and then forwards the amplified signal to the destination via one active transmit antenna, whose index carries the private information of the relay. Specifically, the received signal at the destination can be written as

$$
\mathbf{y}_{D}=A \mathbf{g}_{m} y_{R}+\mathbf{w}_{D}=A \sqrt{P_{S}} h_{l} s \mathbf{g}_{m}+A w_{R} \mathbf{g}_{m}+\mathbf{w}_{D}=A \sqrt{P_{S}} h_{l} s \mathbf{g}_{m}+\tilde{\mathbf{w}}_{D},
$$

where $A$ is the amplification factor, $m$ is the active antenna index of the relay determined by the $b^{R}$ bits, $\tilde{\mathbf{w}}_{D}=A w_{R} \mathbf{g}_{m}+\mathbf{w}_{D}$, and $\mathbf{w}_{D}$ is defined in (5). In this paper, we consider the semi-blind fixed-gain AF relaying assuming knowledge of the average channel gain of the first hop. In this scenario, $A$ can be given by

$$
A=\sqrt{\frac{P_{R}}{P_{S} \Omega_{S R}+N_{0}}} .
$$

It is clear that the covariance matrix of $\tilde{\mathbf{w}}_{D}$ is $\boldsymbol{\Sigma}_{m}=E\left\{\tilde{\mathbf{w}}_{D} \tilde{\mathbf{w}}_{D}^{H}\right\}=A^{2} N_{0} \mathbf{g}_{m} \mathbf{g}_{m}^{H}+N_{0} \mathbf{I}_{N_{r D}}$, and the PDF of $\mathbf{y}_{D}$ can be derived as

$$
f\left(\mathbf{y}_{D}\right)=\frac{1}{\pi^{N_{r D}} \operatorname{det}\left(\boldsymbol{\Sigma}_{m}\right)} \exp \left(-\left(\mathbf{y}_{D}-A \sqrt{P_{S}} h_{l} s \mathbf{g}_{m}\right)^{H} \boldsymbol{\Sigma}_{m}^{-1}\left(\mathbf{y}_{D}-A \sqrt{P_{S}} h_{l} s \mathbf{g}_{m}\right)\right) .
$$

Therefore, the optimal ML detection for $l, s$, and $m$ can be formulated as

$$
\begin{aligned}
(\hat{l}, \hat{s}, \hat{m}) & =\arg \max _{l, s, m} f\left(\mathbf{y}_{D}\right) \\
& =\arg \min _{l, s, m}\left\{\ln \left(\operatorname{det}\left(\boldsymbol{\Sigma}_{m}\right)\right)+\left(\mathbf{y}_{D}-A \sqrt{P_{S}} h_{l} s \mathbf{g}_{m}\right)^{H} \boldsymbol{\Sigma}_{m}^{-1}\left(\mathbf{y}_{D}-A \sqrt{P_{S}} h_{l} s \mathbf{g}_{m}\right)\right\}
\end{aligned}
$$

where $\hat{l}, \hat{s}$, and $\hat{m}$ are the estimates of $l, s$, and $m$, respectively, at the destination. Unfortunately, the matrix inversion that incurs high computational complexity is required for the optimal ML detection in (10). To solve this problem, we resort to the approximation of $\boldsymbol{\Sigma}_{m}$ :

$$
\boldsymbol{\Sigma}_{m} \approx\left(A^{2} \Omega_{R D}+1\right) N_{0} \mathbf{I}_{N_{r D}},
$$


such that (10) reduces to the simplified ML detection as follows:

$$
(\hat{l}, \hat{s}, \hat{m})=\arg \min _{l, s, m}\left\|\mathbf{y}_{D}-A \sqrt{P_{S}} h_{l} s \mathbf{g}_{m}\right\|^{2} .
$$

From (10) or (12), the transmitted information from both the source and relay can be obtained. The performance comparison between (10) and (12) will be given in Section V.

Remark 1: For DF relaying, only local channel state information (CSI) is needed, but the number of the transmit antennas of the relay is required to be no smaller than that of the source. For AF relaying, no restriction is imposed, but global CSI is needed at the destination.

\section{Performance AnAlysis for DF Relaying}

In this section, we analyze the ABEP performance of SMRN-RPI with DF relaying by assuming the optimal ML detection. Tight upper bounds on the ABEP are derived in closed form for the relay and source.

\section{A. ABEP of the Relay}

The relay embeds its information into the index of one active transmit antenna. An error happens once the index of the active antenna is detected incorrectly at the destination as one of the remaining $N_{t R}-1$ indices with equal probability. A different incorrect index may result in a different number of erroneous bits, and we observe that there are in total $\left(\begin{array}{c}b^{R} \\ t\end{array}\right)$ error events that correspond to $t$ erroneous spatial bits [35], where $t \in\left\{1, \ldots, b^{R}\right\}$. Therefore, on average, the ABEP of the relay can be given by

$$
P_{b R}=\frac{P_{I R}}{b^{R}\left(N_{t R}-1\right)} \sum_{t=1}^{b^{R}} t \cdot\left(\begin{array}{c}
b^{R} \\
t
\end{array}\right)=\frac{1}{2} P_{I R} \cdot \frac{N_{t R}}{N_{t R}-1},
$$

where $P_{I R}$ denotes the error probability of detecting the active antenna index $m$. To derive $P_{I R}$, we first investigate the conditional pairwise error probability (PEP) from (4), which is the probability of detecting $\mathbf{x}_{R}$ erroneously as $\hat{\mathbf{x}}_{R}$ conditioned on $\mathbf{G}$, namely

$$
\begin{aligned}
\operatorname{Pr}\left(\mathbf{x}_{R} \rightarrow \hat{\mathbf{x}}_{R} \mid \mathbf{G}\right) & =\operatorname{Pr}\left(\left\|\mathbf{y}_{D}-\sqrt{P_{R}} \mathbf{G} \mathbf{x}_{R}\right\|^{2}>\left\|\mathbf{y}_{D}-\sqrt{P_{R}} \mathbf{G} \hat{\mathbf{x}}_{R}\right\|^{2}\right) \\
& =Q\left(\sqrt{\frac{P_{R} \kappa}{2 N_{0}}}\right),
\end{aligned}
$$


where $\kappa=\left\|\left(\mathbf{g}_{\hat{l}} x^{\Re}+j \mathbf{g}_{m} x^{\Im}\right)-\left(\mathbf{g}_{\hat{l}} \hat{x}^{\Re}+j \mathbf{g}_{\hat{m}} \hat{x}^{\Im}\right)\right\|^{2}=\|\mathbf{a}+j \mathbf{b}\|^{2}$ with

$$
\begin{aligned}
& \mathbf{a}=\Re\left\{\mathbf{g}_{\tilde{l}} x^{\Re}\right\}-\Im\left\{\mathbf{g}_{m} x^{\Im}\right\}-\Re\left\{\mathbf{g}_{\hat{l}} \hat{x}^{\Re}\right\}+\Im\left\{\mathbf{g}_{\hat{m}} \hat{x}^{\Im}\right\}, \\
& \mathbf{b}=\Im\left\{\mathbf{g}_{\tilde{l}} x^{\Re}\right\}+\Re\left\{\mathbf{g}_{m} x^{\Im}\right\}-\Im\left\{\mathbf{g}_{\hat{l}} \hat{x}^{\Re}\right\}-\Re\left\{\mathbf{g}_{\hat{m}} \hat{x}^{\Im}\right\} .
\end{aligned}
$$

We observe from (15) that $\kappa=\sum_{i=1}^{2 N_{r D}} \alpha_{i}^{2}$, where $\alpha_{i} \sim \mathcal{C N}\left(0, \sigma_{1}^{2}\right)$ with

$$
\sigma_{1}^{2}= \begin{cases}\frac{1}{2} \Omega_{R D}\left(\left|x^{\Re}\right|^{2}+\left|\hat{x}^{\Re}\right|^{2}+\left|x^{\Im}\right|^{2}+\left|\hat{x}^{\Im}\right|^{2}\right), & \text { for } \tilde{l} \neq \hat{l} \text { and } m \neq \hat{m} \\ \frac{1}{2} \Omega_{R D}\left(\left|x^{\Re}-\hat{x}^{\Re}\right|^{2}+\left|x^{\Im}\right|^{2}+\left|\hat{x}^{\Im}\right|^{2}\right), & \text { for } \tilde{l}=\hat{l} \text { and } m \neq \hat{m} \\ \frac{1}{2} \Omega_{R D}\left(\left|x^{\Re}\right|^{2}+\left|\hat{x}^{\Re}\right|^{2}+\left|x^{\Im}-\hat{x}^{\Im}\right|^{2}\right), & \text { for } \tilde{l} \neq \hat{l} \text { and } m=\hat{m} \\ \frac{1}{2} \Omega_{R D}\left(\left|x^{\Re}-\hat{x}^{\Re}\right|^{2}+\left|x^{\Im}-\hat{x}^{\Im}\right|^{2}\right), & \text { for } \tilde{l}=\hat{l} \text { and } m=\hat{m} .\end{cases}
$$

Hence, $\kappa$ is chi-squared distributed with $2 N_{r D}$ degrees of freedom, whose PDF is given by [36]

$$
f_{\kappa}(v)=\frac{1}{2^{N_{r D}} \Gamma\left(N_{r D}\right) \sigma_{1}^{2 N_{r D}}} v^{N_{r D}-1} \exp \left(-\frac{v}{2 \sigma_{1}^{2}}\right) .
$$

By averaging $\operatorname{Pr}\left(\mathbf{x}_{R} \rightarrow \hat{\mathbf{x}}_{R} \mid \mathbf{G}\right)$ over $\kappa$, we arrive at [37, Eq. (64)]

$$
\begin{aligned}
\operatorname{Pr}\left(\mathbf{x}_{R} \rightarrow \hat{\mathbf{x}}_{R}\right) & =\int_{0}^{+\infty} Q\left(\sqrt{\frac{P_{R} v}{2 N_{0}}}\right) f_{\kappa}(v) d v \\
& =\left[P\left(c_{1}\right)\right]^{N_{r D}} \sum_{k=0}^{N_{r D}-1}\left(\begin{array}{c}
N_{r D}-1+k \\
k
\end{array}\right)\left[1-P\left(c_{1}\right)\right]^{k},
\end{aligned}
$$

where

$$
P\left(c_{1}\right)=\frac{1}{2}\left(1-\sqrt{\frac{c_{1}}{1+c_{1}}}\right)
$$

with $c_{1}=P_{R} \sigma_{1}^{2} /\left(2 N_{0}\right)$. Let $\operatorname{PEP}_{1}(x, \hat{x})$ denote $\operatorname{Pr}\left(\mathbf{x}_{R} \rightarrow \hat{\mathbf{x}}_{R}\right)$ associated with $\tilde{l} \neq \hat{l}$ and $m \neq \hat{m}$, and $\operatorname{PEP}_{2}(x, \hat{x})$ denote $\operatorname{Pr}\left(\mathbf{x}_{R} \rightarrow \hat{\mathbf{x}}_{R}\right)$ associated with $\tilde{l}=\hat{l}$ and $m \neq \hat{m}$. Then, according to the union bounding technique, an upper bound on the error probability of detecting the active antenna index $m$ at the destination is given by

$$
\begin{aligned}
P_{I R} & \leq \frac{1}{N_{t R} N_{t S} M} \sum_{\substack{m, \hat{m} \\
m \neq \hat{m}}} \sum_{\tilde{l}, \hat{l}} \sum_{x, \hat{x}} \operatorname{Pr}\left(\mathbf{x}_{R} \rightarrow \hat{\mathbf{x}}_{R}\right) \\
& =\frac{1}{N_{t R} N_{t S} M}\left(\sum_{\substack{m, \hat{m} \\
m \neq \hat{m}}} \sum_{\substack{\tilde{l}, \hat{l} \\
l \neq \hat{l}}} \sum_{x, \hat{x}} \operatorname{Pr}\left(\mathbf{x}_{R} \rightarrow \hat{\mathbf{x}}_{R}\right)+\sum_{\substack{m, \hat{m} \\
m \neq \hat{m}}} \sum_{\substack{\tilde{l} \hat{l} \\
\tilde{l}=\hat{l}}} \sum_{x, \hat{x}} \operatorname{Pr}\left(\mathbf{x}_{R} \rightarrow \hat{\mathbf{x}}_{R}\right)\right) \\
& =\frac{\left(N_{t R}-1\right)\left(N_{t S}-1\right)}{M} \sum_{x, \hat{x}} \operatorname{PEP}_{1}(x, \hat{x})+\frac{N_{t R}-1}{M} \sum_{x, \hat{x}} \operatorname{PEP}_{2}(x, \hat{x}) .
\end{aligned}
$$


Finally, substituting (20) into (13) yields an upper bound on the ABEP of the relay, namely

$$
P_{b R} \leq \frac{N_{t R}\left(N_{t S}-1\right)}{2 M} \sum_{x, \hat{x}} \operatorname{PEP}_{1}(x, \hat{x})+\frac{N_{t R}}{2 M} \sum_{x, \hat{x}} \operatorname{PEP}_{2}(x, \hat{x}) .
$$

It is clearly shown in (21) that the ABEP performance of the relay becomes worse with increasing $N_{t S}$ and $N_{t R}$. This is understandable as the ABEP performance of the relay becomes worse with the increase of the data rate of the relay, namely the increase of $N_{t R}$. The worse ABEP performance with increasing $N_{t S}$ can be accounted for its close relationship with $N_{t R}$, namely $N_{t R} \geq N_{t S}$

\section{B. ABEP of the Source}

As described in Section II, the information transmitted from the source is split into spatial bits and constellation bits. This feature motivates us to divide the total ABEP of the source, denoted by $P_{b S}$, into two parts: the ABEP for the symbol bits, denoted by $P_{1 S}$, and the ABEP for the spatial bits, denoted by $P_{2 S}$. Obviously, we have

$$
P_{b S}=\frac{b_{1}^{S} P_{1 S}+b_{2}^{S} P_{2 S}}{b_{1}^{S}+b_{2}^{S}} .
$$

Note that error propagation occurs at the relay, which should be taken into account. Let us first consider the derivation of $P_{2 S}$. Similar to (13), $P_{2 S}$ can be expressed as

$$
P_{2 S}=\frac{1}{2} P_{I S} \cdot \frac{N_{t S}}{N_{t S}-1},
$$

where $P_{I S}$ is the error probability of detecting the active antenna index $l$ at the destination. By neglecting the small probability event that an erroneous estimation of $l$ at the relay is then "erroneously" estimated to the correct one, $P_{I S}$ can be upper bounded by

$$
P_{I S} \leq 1-\left(1-P_{I_{1} S}\right)\left(1-P_{I_{2} S}\right)
$$

where $P_{I_{1} S}$ denotes the error probability of detecting the active antenna index $l$ in the first hop, and $P_{I_{2} S}$ denotes the error probability of detecting the active antenna index $\tilde{l}$ in the second hop. Similar to (20), $P_{I_{2} S}$ can be easily derived as

$$
\begin{aligned}
P_{I_{2} S} & \leq \frac{1}{N_{t R} N_{t S} M} \sum_{\substack{\hat{\imath}, \hat{l} \\
\hat{l} \neq \hat{l}}} \sum_{m, \hat{m}} \sum_{x, \hat{x}} \operatorname{Pr}\left(\mathbf{x}_{R} \rightarrow \hat{\mathbf{x}}_{R}\right) \\
& =\frac{\left(N_{t R}-1\right)\left(N_{t S}-1\right)}{M} \sum_{x, \hat{x}} \operatorname{PEP}_{1}(x, \hat{x})+\frac{N_{t S}-1}{M} \sum_{x, \hat{x}} \operatorname{PEP}_{3}(x, \hat{x}),
\end{aligned}
$$


where $\operatorname{PEP}_{3}(x, \hat{x})$ denotes $\operatorname{Pr}\left(\mathbf{x}_{R} \rightarrow \hat{\mathbf{x}}_{R}\right)$ associated with $\tilde{l} \neq \hat{l}$ and $m=\hat{m}$. For the calculation of $P_{I_{1} S}$, we study the conditional PEP in the first hop from (3), which is the probability of detecting $\mathbf{x}_{S}$ erroneously as $\tilde{\mathbf{x}}_{S}$ conditioned on $\mathbf{h}$, namely

$$
\operatorname{Pr}\left(\mathbf{x}_{S} \rightarrow \tilde{\mathbf{x}}_{S} \mid \mathbf{h}\right)=\operatorname{Pr}\left(\left|y_{R}-\sqrt{P_{S}} h_{l} s\right|^{2}>\left|y_{R}-\sqrt{P_{S}} h_{\tilde{l}} \tilde{s}\right|^{2}\right)=Q\left(\sqrt{\frac{P_{S} \varsigma}{2 N_{0}}}\right),
$$

where $\varsigma=\left|h_{l} s-h_{\tilde{l}} \tilde{s}\right|^{2}$. Obviously, $\varsigma$ is an exponential random variable, whose PDF is

$$
f_{\varsigma}(v)=\frac{1}{2 \sigma_{2}^{2}} \exp \left(-\frac{v}{2 \sigma_{2}^{2}}\right)
$$

with

$$
\sigma_{2}^{2}= \begin{cases}\Omega_{S R}, & \text { for } \tilde{l} \neq l \\ \frac{1}{2} \Omega_{S R}|s-\tilde{s}|^{2}, & \text { for } \tilde{l}=l\end{cases}
$$

Averaging $\operatorname{Pr}\left(\mathbf{x}_{S} \rightarrow \tilde{\mathbf{x}}_{S} \mid \mathbf{h}\right)$ over $\varsigma$ produces

$$
\operatorname{Pr}\left(\mathbf{x}_{S} \rightarrow \tilde{\mathbf{x}}_{S}\right)=\frac{1}{2}\left(1-\sqrt{\frac{c_{2}}{1+c_{2}}}\right),
$$

where $c_{2}=P_{S} \sigma_{2}^{2} /\left(2 N_{0}\right)$. Therefore, we have

$$
P_{I_{1} S} \leq \frac{1}{N_{t S} M} \sum_{\substack{l, \tilde{l} \\ l \neq \tilde{l}}} \sum_{s, \tilde{s}} \operatorname{Pr}\left(\mathbf{x}_{S} \rightarrow \tilde{\mathbf{x}}_{S}\right)=\frac{1}{2} M\left(N_{t S}-1\right)\left(1-\sqrt{\frac{c_{2}}{1+c_{2}}}\right) .
$$

By substituting (24), (25), and (30) into (23), we obtain $P_{2 S}$.

Next, we turn to the deduction of $P_{1 S}$. It can be shown that an upper bound on $P_{1 S}$ is given by

$$
P_{1 S} \leq 1-\left(1-P_{1 S-S R}\right)\left(1-P_{1 S-R D}\right)
$$

where $P_{1 S-S R}$ and $P_{1 S-R D}$ denote the ABEPs for the symbol bits in the first and second hops, respectively. Obviously, the calculations of both $P_{1 S-S R}$ and $P_{1 S-R D}$ can be classified into two complementary types of error events, depending on whether the indices of the active antennas are detected correctly or not. Specifically, $P_{1 S-S R}$ can be easily evaluated as

$$
P_{1 S-S R}=\frac{1}{2} P_{I_{1} S}+\left(1-P_{I_{1} S}\right) P_{b-\mathrm{PSK}}
$$

where the factor $1 / 2$ results from the fact that there is no constellation symbol transmitted from inactive antennas, $P_{I_{1} S}$ is given by (30), and $P_{b-\mathrm{PSK}}$ is the ABEP of $M$-PSK over a Rayleigh 
fading channel. Note that the instantaneous ABEP of $M$-PSK can be well approximated as [38, Eq. (8.32)]

$$
P_{s b \mid \rho_{S}} \cong \frac{2}{\max \left(\log _{2}(M), 2\right)} \sum_{i=1}^{\max (M / 4,1)} Q\left(\sqrt{2 \rho_{S} \sin ^{2}\left(\frac{(2 i-1) \pi}{M}\right)}\right),
$$

where $\rho_{S}=P_{S}\left|h_{l}\right|^{2} / N_{0}$. Averaging (33) over $\rho_{S}$ yields

$$
P_{b-\mathrm{PSK}} \cong \frac{2}{\max \left(\log _{2}(M), 2\right)} \sum_{i=1}^{\max (M / 4,1)} \frac{1}{2}\left(1-\sqrt{\frac{g_{\mathrm{PSK}}}{1+g_{\mathrm{PSK}}}}\right),
$$

where $g_{\mathrm{PSK}}=P_{S} \Omega_{S R} \sin ^{2}((2 i-1) \pi / M) / N_{0}$. For the derivation of $P_{1 S-R D}$, similar to (32), we have

$$
P_{1 S-R D} \approx \frac{1}{2} P_{\tilde{l} m}+\left(1-P_{\tilde{l} m}\right) P_{b-\mathrm{QAM}}
$$

where $P_{\tilde{l} m}$ denotes the probability of detecting $\tilde{l}$ or $m$ erroneously, and $P_{b-\mathrm{QAM}}$ is the ABEP of $M$-QAM over $N_{r D}$ i.i.d. Rayleigh fading channels. Note that $M$-ary QAM consists of two independent pulse amplitude modulation (PAM) signals, namely $M_{I}$-ary PAM of the I- signal and $M_{Q}$-ary PAM of the Q- signal. As a result, $P_{b-\mathrm{QAM}}$ can be derived as [22]

$$
P_{b-\mathrm{QAM}}=\frac{1}{\log _{2}(M)}\left(\sum_{m=1}^{\log _{2}\left(M_{I}\right)} P_{b I}(m)+\sum_{m=1}^{\log _{2}\left(M_{Q}\right)} P_{b Q}(m)\right)
$$

where

$$
P_{b I / b Q}(m)=\frac{2}{M_{I / Q}} \sum_{i=0}^{\left(1-2^{-m}\right) M_{I / Q}-1}\left\{(-1)^{\left\lfloor\frac{i \cdot 2^{m-1}}{M_{I / Q}}\right\rfloor}\left(2^{m-1}-\left\lfloor\frac{i \cdot 2^{m-1}}{M_{I / Q}}+\frac{1}{2}\right\rfloor\right) \cdot T(i)\right\},
$$

with

$$
\begin{gathered}
T(i)=\left(\frac{1-\mu_{c}(i)}{2}\right)^{N_{r D}} \sum_{k=0}^{N_{r D}-1}\left(\begin{array}{c}
N_{r D}-1+k \\
k
\end{array}\right)\left(\frac{1+\mu_{c}(i)}{2}\right)^{k}, \\
\mu_{c}(i)=\sqrt{\frac{\bar{\rho}_{R}(2 i+1)^{2} d^{2}}{1+\bar{\rho}_{R}(2 i+1)^{2} d^{2}}},
\end{gathered}
$$

$d=\sqrt{3 /\left(M_{I}^{2}+M_{Q}^{2}-2\right)}$, and $\bar{\rho}_{R}=P_{R} \Omega_{R D} / N_{0}$. On the other hand, similar to (20), $P_{l_{m}}$ can be easily obtained from (18) as

$$
\begin{aligned}
P_{\tilde{l} m} \leq & \frac{\left(N_{t R}-1\right)\left(N_{t S}-1\right)}{M} \sum_{x, \hat{x}} \operatorname{PEP}_{1}(x, \hat{x})+\frac{N_{t R}-1}{M} \sum_{x, \hat{x}} \operatorname{PEP}_{2}(x, \hat{x}) \\
& +\frac{N_{t S}-1}{M} \sum_{x, \hat{x}} \operatorname{PEP}_{3}(x, \hat{x}) .
\end{aligned}
$$


From (31)-(40), we can obtain $P_{1 S}$. Finally, after obtaining $P_{1 S}$ and $P_{2 S}$, a closed-form upper bound on the ABEP of the source can be derived from (22).

Remark 2: By taking the Taylor series of the exponential function and ignoring higher order terms, (17) can be rewritten as

$$
f_{\kappa}(v) \approx \frac{1}{2^{N_{r D}} \Gamma\left(N_{r D}\right) \sigma_{1}^{2 N_{r D}}} v^{N_{r D}-1} .
$$

Substituting (41) into (18) yields the following asymptotic unconditional PEP:

$$
\operatorname{Pr}\left(\mathbf{x}_{R} \rightarrow \hat{\mathbf{x}}_{R}\right)=\frac{2^{N_{r D}-1} \Gamma\left(N_{r D}+0.5\right)}{\sqrt{\pi}\left(2 N_{r D}\right) ! !}\left(\frac{1}{c_{1}}\right)^{N_{r D}},
$$

which achieves a diversity order of $N_{r D}$. Hence, the diversity orders of $P_{I R}$ in (20), $P_{I_{2} S}$ in (25), and $P_{\tilde{l}_{m}}$ in (40) are all governed by $N_{r D}$. By contrast, since there is only a single receive antenna at the relay, we observe from (23)-(24) and (30)-(34) that both $P_{1 S}$ and $P_{2 S}$ achieve the same diversity order of unity. To sum up, the diversity orders of the relay and source are given by $N_{r D}$ and 1 , respectively.

Remark 3: Due to the higher diversity order, $P_{I_{2} S}$ in (24) and $P_{\tilde{l} m}$ in (35) are relatively small at high SNR with $N_{r D}>1$. Therefore, the asymptotic performance of the source with $N_{r D}>1$ can be easily derived by setting $P_{I_{2} S}=0$ and $P_{\tilde{l}_{m}}=0$, which implies that the asymptotic performance of the source is independent of $N_{t R}$ when $N_{r D}>1$.

\section{Performance Analysis for AF RElaying}

Since the error probability of the optimal ML detector in (10) is extremely difficult to evaluate, in this section, we concentrate on the performance analysis of the simplified ML detector for SMRN-RPI with AF relaying. After the derivation of PEP in closed form, we provide upper bounds on the ABEPs of the source and relay.

\section{A. PEP Analysis}

From (12), the conditional PEP, which is the probability of detecting $(l, s, m)$ incorrectly as $(\hat{l}, \hat{s}, \hat{m})$, can be expressed as

$$
\begin{aligned}
\operatorname{Pr}((l, s, m) & \rightarrow(\hat{l}, \hat{s}, \hat{m}) \mid \mathbf{h}, \mathbf{G})=\operatorname{Pr}\left(\left\|\mathbf{y}_{D}-A \sqrt{P_{S}} h_{l} s \mathbf{g}_{m}\right\|^{2}>\left\|\mathbf{y}_{D}-A \sqrt{P_{S}} h_{\hat{l}} \hat{s} \mathbf{g}_{\hat{m}}\right\|^{2}\right) \\
& =\operatorname{Pr}\left(-A^{2} P_{S}\left\|h_{l} s \mathbf{g}_{m}-h_{\hat{l}} \hat{s} \mathbf{g}_{\hat{m}}\right\|^{2}-2 A \sqrt{P_{S}} \Re\left\{\tilde{\mathbf{w}}_{D}^{H}\left(h_{l} s \mathbf{g}_{m}-h_{\hat{l}} \hat{s} \mathbf{g}_{\hat{m}}\right)\right\}>0\right) \\
& =\operatorname{Pr}(D>0) .
\end{aligned}
$$


It can be shown that $D$ is a Gaussian random variable with

$$
E\{D\}=-A^{2} P_{S}\left\|h_{l} s \mathbf{g}_{m}-h_{\hat{l}} \hat{s} \mathbf{g}_{\hat{m}}\right\|^{2}, \quad \operatorname{Var}\{D\}=2 N_{0} A^{2} P_{S}\left\|\mathbf{C}\left(h_{l} s \mathbf{g}_{m}-h_{\hat{l}} \hat{s} \mathbf{g}_{\hat{m}}\right)\right\|^{2},
$$

where $\mathbf{C}=\operatorname{diag}\left\{\left[\sqrt{A^{2}\left|g_{1 m}\right|^{2}+1}, \ldots, \sqrt{A^{2}\left|g_{N_{r D} m}\right|^{2}+1}\right]^{T}\right\}$. Hence, (43) can be rewritten as

$$
\operatorname{Pr}((l, s, m) \rightarrow(\hat{l}, \hat{s}, \hat{m}) \mid \mathbf{h}, \mathbf{G})=Q\left(\frac{A^{2} P_{S}\left\|h_{l} s \mathbf{g}_{m}-h_{\hat{l}} \hat{s} \mathbf{g}_{\hat{m}}\right\|^{2}}{\sqrt{2 N_{0} A^{2} P_{S}\left\|\mathbf{C}\left(h_{l} s \mathbf{g}_{m}-h_{\hat{l}} \hat{s} \mathbf{g}_{\hat{m}}\right)\right\|^{2}}}\right)
$$

In order to obtain the unconditional PEP, (45) should be averaged over $\mathbf{h}$ and $\mathbf{G}$. However, it is not an easy task to derive a closed-form expression due to the complexity of (45). Therefore, we consider the case of $N_{r D}=1$ here. Later in Section V, we will employ numerical simulations to evaluate the performance for the case of $N_{r D}>1$.

With $N_{r D}=1$, (45) reduces to

$$
\operatorname{Pr}((l, s, m) \rightarrow(\hat{l}, \hat{s}, \hat{m}) \mid \mathbf{h}, \mathbf{g})=Q\left(\sqrt{\frac{\rho\left|h_{l} s g_{m}-h_{\hat{l}} \hat{s} g_{\hat{m}}\right|^{2}}{2\left(\left|g_{m}\right|^{2}+C\right)}}\right),
$$

where $C=1 / A^{2}$. By using the following approximation of the Gaussian $Q$-function:

$$
Q(v) \approx \frac{1}{12} \exp \left(-\frac{v^{2}}{2}\right)+\frac{1}{4} \exp \left(-\frac{2 v^{2}}{3}\right)
$$

(46) becomes

$$
\operatorname{Pr}((l, s, m) \rightarrow(\hat{l}, \hat{s}, \hat{m}) \mid \mathbf{h}, \mathbf{g}) \approx \frac{1}{12} \exp \left(-\frac{\rho}{4} \lambda\right)+\frac{1}{4} \exp \left(-\frac{\rho}{3} \lambda\right)
$$

where

$$
\lambda=\frac{\left|h_{l} s g_{m}-h_{\hat{l}} \hat{s} g_{\hat{m}}\right|^{2}}{\left|g_{m}\right|^{2}+C} .
$$

Depending on the relationships between $m$ and $\hat{m}$, and between $l$ and $\hat{l}, \lambda$ evolves into four specific variables as follows:

$$
\begin{cases}\lambda_{1}=\frac{\left|g_{m}\right|^{2}\left|h_{l}\right|^{2}|s-\hat{s}|^{2}}{\left|g_{m}\right|^{2}+C}, & \text { for } m=\hat{m} \text { and } l=\hat{l} \\ \lambda_{2}=\frac{\left|g_{m}\right|^{2}\left|h_{l} s-h_{\hat{l}} \hat{s}\right|^{2}}{\left|g_{m}\right|^{2}+C}, & \text { for } m=\hat{m} \text { and } l \neq \hat{l} \\ \lambda_{3}=\frac{\left|h_{l}\right|^{2}\left|g_{m} s-g_{\hat{m}} \hat{s}\right|^{2}}{\left|g_{m}\right|^{2}+C}, & \text { for } m \neq \hat{m} \text { and } l=\hat{l} \\ \lambda_{4}=\frac{\left|h_{l} s g_{m}-h_{\hat{s}} \hat{s} g_{\hat{m}}\right|^{2}}{\left|g_{m}\right|^{2}+C}, & \text { for } m \neq \hat{m} \text { and } l \neq \hat{l} .\end{cases}
$$

Accordingly, the overall error events can also be classified into four types. Let us define the unconditional PEP for the first type as $\mathrm{PEP}_{1}$, the second type as $\mathrm{PEP}_{2}$, the third type as $\mathrm{PEP}_{3}$, 
and the fourth type as $\mathrm{PEP}_{4}$. Obviously, $\mathrm{PEP}_{i}$ is associated with $\lambda_{i}$, and the moment generating function (MGF) of $\lambda_{i}$ is required for obtaining $\operatorname{PEP}_{i}$, where $i \in\{1,2,3,4\}$. The following propositions outline the MGFs of $\lambda_{i}, i=1,2,3,4$.

Proposition 1: The MGFs of $\lambda_{1}$ and $\lambda_{2}$ are given by

$$
\mathcal{M}_{\lambda_{i}}(t)=\frac{C}{\Omega_{R D} \omega_{i}(t)}\left(1-\frac{1}{\omega_{i}(t)}\right) \exp \left(\frac{C}{\Omega_{R D} \omega_{i}(t)}\right) \mathrm{E}_{1}\left(\frac{C}{\Omega_{R D} \omega_{i}(t)}\right)+\frac{1}{\omega_{i}(t)}, \quad i=1,2
$$

where $\omega_{1}(t)=1-|s-\hat{s}|^{2} \Omega_{S R} t$ and $\omega_{2}(t)=1-2 \Omega_{S R} t$.

Proof: See Appendix A.

Proposition 2: The MGF of $\lambda_{3}$ can be evaluated as

$$
\begin{aligned}
\mathcal{M}_{\lambda_{3}}(t) \approx & \frac{\Omega_{S R}}{2}+\frac{1}{8 \Omega_{S R}^{2} t^{3}}{ }_{3} F_{2}\left(1,2,5 / 2 ; 3 / 2,5 / 2 ;-\frac{1}{4 \Omega_{S R}^{2} t^{2}}\right) \\
& +t\left(\frac{1}{2 \Omega_{S R}^{2}}+2 t^{2}\right)^{-1} Q_{1}^{-1}\left(-t\left(\frac{1}{4 \Omega_{S R}^{2}}+t^{2}\right)^{-1 / 2}\right) \\
& -\frac{1}{4 t}+\frac{1}{4 \Omega_{S R} t^{2}}{ }_{3} F_{2}\left(1,3 / 2,2 ; 3 / 2,3 / 2 ;-\frac{1}{4 \Omega_{S R}^{2} t^{2}}\right) \\
& -t\left(\frac{1}{\Omega_{S R}^{2}}+4 t^{2}\right)^{-1} Q_{1}^{0}\left(-t\left(\frac{1}{4 \Omega_{S R}^{2}}+t^{2}\right)^{-1 / 2}\right)
\end{aligned}
$$

for $P_{S} \Omega_{S R} \ll P_{R} \Omega_{R D}$, while

$$
\mathcal{M}_{\lambda_{3}}(t) \approx-\frac{C}{2 \Omega_{S R} \Omega_{R D} t} \exp \left(-\frac{C}{2 \Omega_{S R} \Omega_{R D} t}\right) \mathrm{E}_{1}\left(-\frac{C}{2 \Omega_{S R} \Omega_{R D} t}\right)
$$

for $P_{S} \Omega_{S R} \gg P_{R} \Omega_{R D}$.

Proof: See Appendix B.

Since the derivation of the MGF of $\lambda_{4}$ involves an integral that includes the exponential integral function, i.e., $\mathrm{E}_{1}(\cdot)$, before presenting Proposition 3, we propose the following lemma for an accurate approximation of $\mathrm{E}_{1}(\cdot)$.

Lemma: $\mathrm{E}_{1}(v)$ can be approximated as

$$
\mathrm{E}_{1}(v) \approx 4 \pi \sum_{i=1}^{2} \sum_{k=1}^{2} p_{i} p_{k} \sqrt{q_{i}} \exp \left(-q_{i} q_{k} v\right),
$$

where $p_{1}=1 / 12, p_{2}=1 / 4, q_{1}=1$, and $q_{2}=4 / 3$.

Proof: The definition of $\mathrm{E}_{1}(v)$ can be rewritten as

$$
\mathrm{E}_{1}(v)=\int_{v}^{\infty}\left(\frac{\exp (-t)}{\sqrt{t}}\right)\left(\frac{1}{\sqrt{t}}\right) d t, \quad v>0 .
$$


It can be observed that [39]

$$
\frac{\exp (-t)}{\sqrt{t}}=-2 \sqrt{\pi} \frac{d}{d t}\{Q(\sqrt{2 t})\} .
$$

Besides, resorting to the approximation of the Gaussian $Q$-function in (47), we have

$$
Q(\sqrt{2 t}) \approx \sum_{i=1}^{2} p_{i} \exp \left(-q_{i} t\right),
$$

where $p_{1}=1 / 12, p_{2}=1 / 4, q_{1}=1$, and $q_{2}=4 / 3$. By using (56) and (57), (55) can be expressed as

$$
\mathrm{E}_{1}(v) \approx 4 \pi \sum_{i=1}^{2} p_{i} \sqrt{q_{i}} Q\left(\sqrt{2 q_{i} v}\right) .
$$

Applying (57) to (58) produces (54), which completes the proof.

Proposition 3: The MGF of $\lambda_{4}$ can be approximated as

$$
\begin{aligned}
\mathcal{M}_{\lambda_{4}}(t) \approx & -\frac{4 \pi}{\Omega_{S R} \Omega_{R D}^{2} t} \sum_{i=1}^{2} \sum_{k=1}^{2} p_{i} p_{k} \sqrt{q_{i}} \exp \left(\frac{\left(q_{i} q_{k}-1\right) C}{\Omega_{S R} \Omega_{R D} t}\right) \\
& \times\left[\left(\frac{\Omega_{S R} \Omega_{R D} t}{\left(q_{i} q_{k}-1\right)\left(1-\Omega_{S R} t\right)-\Omega_{S R} t}\right)^{2}-\frac{C \Omega_{S R} \Omega_{R D} t}{\left(q_{i} q_{k}-1\right)\left(1-\Omega_{S R} t\right)-\Omega_{S R} t}\right],
\end{aligned}
$$

where $p_{1}, p_{2}, q_{1}$, and $q_{2}$ are given in the Lemma.

Proof: See Appendix C.

After obtaining $\mathcal{M}_{\lambda_{i}}(t), \mathrm{PEP}_{i}$ can be readily derived from (48) as

$$
\mathrm{PEP}_{i} \approx \frac{1}{12} \mathcal{M}_{\lambda_{i}}\left(-\frac{\rho}{4}\right)+\frac{1}{4} \mathcal{M}_{\lambda_{i}}\left(-\frac{\rho}{3}\right), \quad i=1,2,3,4 .
$$

\section{B. ABEPs of the Source and the Relay}

According to the union bounding technique, the error probability of detecting the active antenna index $m$ can be evaluated as

$$
\begin{aligned}
& P_{I R} \leq \frac{1}{N_{t S} N_{t R} M}\left(\sum_{\substack{m, \hat{m} \\
m \neq \hat{m}}} \sum_{\substack{l, \hat{l} \\
l=\hat{l}}} \sum_{s, \hat{s}} \operatorname{Pr}((l, s, m) \rightarrow(\hat{l}, \hat{s}, \hat{m}))\right. \\
&\left.\quad+\sum_{\substack{m, \hat{m} \\
m \neq \hat{m}}} \sum_{\substack{l, \hat{l} \\
l \neq \hat{l}}} \sum_{s, \hat{s}} \operatorname{Pr}((l, s, m) \rightarrow(\hat{l}, \hat{s}, \hat{m}))\right) \\
&=M\left(N_{t R}-1\right) \cdot \operatorname{PEP}_{3}+M\left(N_{t R}-1\right)\left(N_{t S}-1\right) \cdot \mathrm{PEP}_{4} .
\end{aligned}
$$


Then, putting (61) into (13) generates an upper bound on the ABEP of the relay as follows:

$$
P_{b R} \leq \frac{1}{2} M N_{t R} \cdot \mathrm{PEP}_{3}+\frac{1}{2} M N_{t R}\left(N_{t S}-1\right) \cdot \mathrm{PEP}_{4} .
$$

Similar to the case of DF relaying, the ABEP of the source can also be expressed as (22), which is rewritten here for reference.

$$
P_{b S}=\frac{b_{1}^{S} P_{1 S}+b_{2}^{S} P_{2 S}}{b_{1}^{S}+b_{2}^{S}},
$$

where $P_{2 S}$ is given in (23), namely

$$
P_{2 S}=\frac{1}{2} P_{I S} \cdot \frac{N_{t S}}{N_{t S}-1} .
$$

Note that the calculations of $P_{2 S}\left(P_{I S}\right)$ and $P_{1 S}$ for AF relaying are different from those for DF relaying. For $P_{I S}$, which is the error probability of detecting the active antenna index $l$, similar to $(61)$, we have

$$
\begin{aligned}
P_{I S} \leq \frac{1}{N_{t S} N_{t R} M}\left(\sum_{\substack{m, \hat{m} \\
m=\hat{m}}} \sum_{\substack{l, \hat{l} \\
l \neq \hat{l}}} \sum_{s, \hat{s}} \operatorname{Pr}((l, s, m) \rightarrow(\hat{l}, \hat{s}, \hat{m}))\right. \\
\left.\quad+\sum_{\substack{m, \hat{m} \\
m \neq \hat{m}}} \sum_{\substack{l, \hat{l} \\
l \neq \hat{l}}} \sum_{s, \hat{s}} \operatorname{Pr}((l, s, m) \rightarrow(\hat{l}, \hat{s}, \hat{m}))\right) \\
=M\left(N_{t S}-1\right) \cdot \operatorname{PEP}_{2}+M\left(N_{t R}-1\right)\left(N_{t S}-1\right) \cdot \mathrm{PEP}_{4} .
\end{aligned}
$$

By substituting (65) into (64), we arrive at

$$
P_{2 S} \leq \frac{1}{2} M N_{t S} \cdot \mathrm{PEP}_{2}+\frac{1}{2} M N_{t S}\left(N_{t R}-1\right) \cdot \mathrm{PEP}_{4} .
$$

It is clear that $P_{1 S}$, i.e., the ABEP for the symbol bits, is related to the detection of $l$ and $m$. Let $P_{l m}$ denote the probability of detecting $l$ or $m$ erroneously. Obviously, we have

$$
P_{l m} \leq M\left(N_{t S}-1\right) \cdot \mathrm{PEP}_{2}+M\left(N_{t R}-1\right) \cdot \mathrm{PEP}_{3}+M\left(N_{t S}-1\right)\left(N_{t R}-1\right) \cdot \mathrm{PEP}_{4} .
$$

When $l$ or $m$ is detected incorrectly at the destination, the ABEP for the $M$-PSK symbol is given by $1 / 2$ since there is no constellation symbol transmitted from inactive antennas. On the other hand, when both $l$ and $m$ are detected correctly, the instantaneous end-to-end SNR is

$$
\rho_{s}=\frac{\rho\left|h_{l}\right|^{2}\left|g_{m}\right|^{2}}{\left|g_{m}\right|^{2}+C},
$$


whose MGF is derived in [40] as

$$
\mathcal{M}_{\rho_{s}}(t)=\frac{1}{1-\rho \Omega_{S R} t}-\frac{C \rho \Omega_{S R} t}{\Omega_{R D}\left(1-\rho \Omega_{S R} t\right)^{2}} \exp \left(\frac{C}{\Omega_{R D}\left(1-\rho \Omega_{S R} t\right)}\right) \mathrm{E}_{1}\left(\frac{C}{\Omega_{R D}\left(1-\rho \Omega_{S R} t\right)}\right) \text {. }
$$

Recalling the conditional ABEP of $M$-PSK demodulation in (33) and the approximation of the Gaussian $Q$-function in (47), we obtain the unconditional ABEP of $M$-PSK demodulation as

$$
\begin{aligned}
P_{b-\mathrm{PSK}} \approx \frac{2}{\max \left(\log _{2}(M), 2\right)} \sum_{i=1}^{\max (M / 4,1)} & \frac{1}{12} \mathcal{M}_{\rho_{s}}\left(-\sin ^{2}\left(\frac{(2 i-1) \pi}{M}\right)\right) \\
+ & \frac{1}{4} \mathcal{M}_{\rho_{s}}\left(-\frac{4}{3} \sin ^{2}\left(\frac{(2 i-1) \pi}{M}\right)\right) .
\end{aligned}
$$

Therefore, $P_{1 S}$ can be obtained as

$$
P_{1 S}=\frac{1}{2} P_{l m}+\left(1-P_{l m}\right) P_{b-\mathrm{PSK}}
$$

By substituting (64) and (71) into (63), we obtain the ABEP of the source.

Remark 4: We observe from (62) that the ABEP of the relay becomes larger with increasing $N_{t S}, N_{t R}$, and $M$. For the source, increasing $N_{t S}$ and $N_{t R}$ results in larger values of $P_{1 S}$ and $P_{2 S}$ according to (66) and (71). Hence, it can be predicted the BER performance of SMRN-RPI deteriorates with increasing $N_{t S}, N_{t R}$, and $M$.

\section{Simulation Results}

In this section, we conduct Monte Carlo simulations to evaluate the uncoded BER performance of SMRN-RPI with DF and AF relaying. In all simulations, the channels are assumed to be Rayleigh flat fading channels and the CSI is perfectly known to the relay and destination. The path-loss exponent is $\alpha=3$. We also consider that $P_{S}=P_{R}$, and plot the BER versus $\rho=P_{S} / N_{0}$ unless otherwise stated. SMRN with unique factorable constellation pair (SMRN-UFCP) and the typical dual-hop SM without relay information are chosen as benchmarks for comparison. In SMRN-UFCP, symbols transmitted by the source are selected from one part of a given UFCP [41] and the relay's symbols are specially selected from the other part of the identical UFCP. Specifically, for DF relaying, with $N_{t S}=N_{t R}$, the relay activates one antenna according to the decoded spatial bits, to transmit the combined symbol that includes the symbol bits from the source and relay; for AF relaying, with $N_{t R}=1$, the relay multiplies the received signal by its own symbol and transmits the resulting signal to the destination. For brevity, we will 


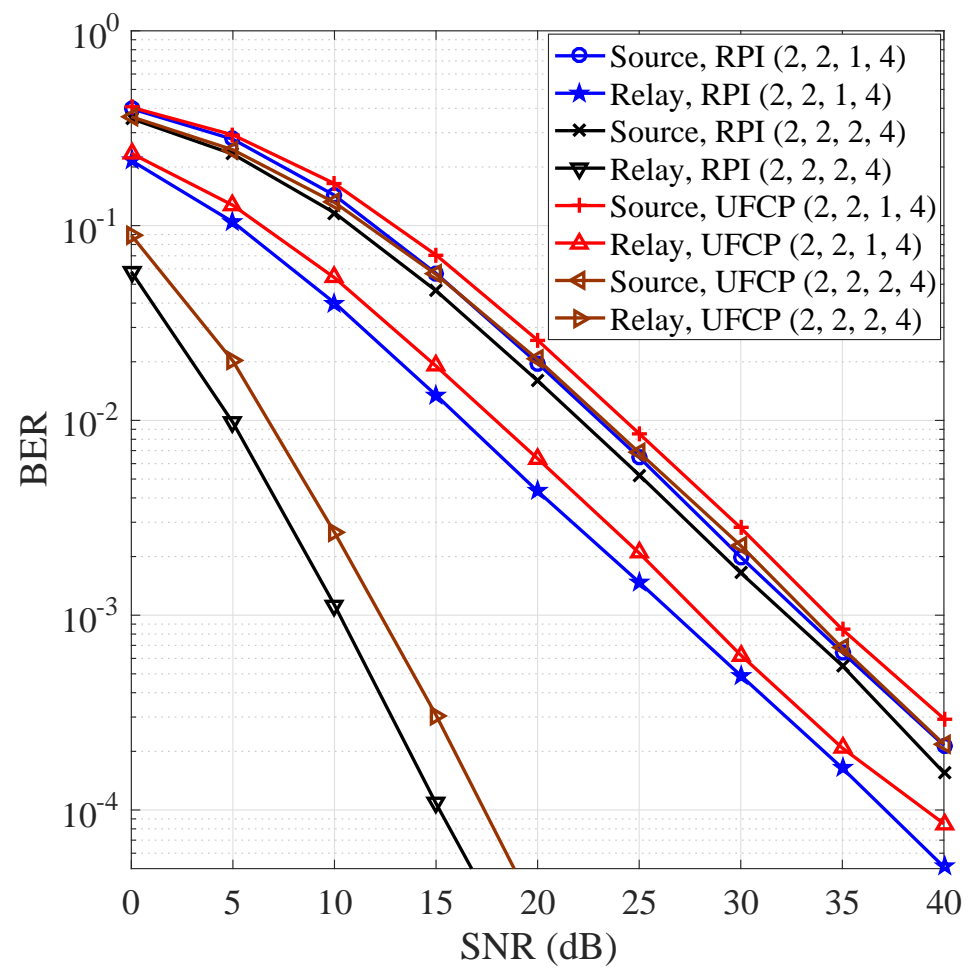

Fig. 2. Performance comparison among "RPI/UFCP $(2,2,1,4)$ " and "RPI/UFCP $(2,2,2,4)$ " with DF relaying, where $d_{S R}=1$ and $d_{R D}=0.5$.

refer to "(w/o) RPI $\left(N_{t S}, N_{t R}, N_{r D}, M\right)$ " as the (dual-hop SM) SMRN-RPI scheme in which the source employs $M$-PSK with $N_{t S}$ transmit antennas, the relay has $N_{t R}$ transmit antennas, and the destination is equipped with $N_{r D}$ receive antennas, and "UFCP $\left(N_{t S}, M_{2}, N_{r D}, M_{1}\right)$ " as the SMRN-UFCP scheme in which the source employs $M_{1}$-ary constellation with $N_{t S}$ transmit antennas, the relay employs $M_{2}$-ary constellation with $N_{t S}$ (for DF relaying) or one (for $\mathrm{AF}$ relaying) transmit antenna, and the destination is equipped with $N_{r D}$ receive antennas.

\section{A. DF Relaying}

In this subsection, the BER performance of SMRN-RPI with DF relaying is evaluated by assuming that both the relay and destination employ ML detection. In Fig. 2, we compare the BER performance of "RPI/UFCP $(2,2,1,4)$ " and "RPI/UFCP $(2,2,2,4)$ " with DF relaying, where $d_{S R}=1$ and $d_{R D}=0.5$. The UFCP for SMRN-UFCP includes $\{1+3 j,-1-3 j,-1+j, 1-j\} / \sqrt{6}$ and $\{1, j\}$ that are employed by the source and relay, respectively. As seen from Fig. 2, with the 


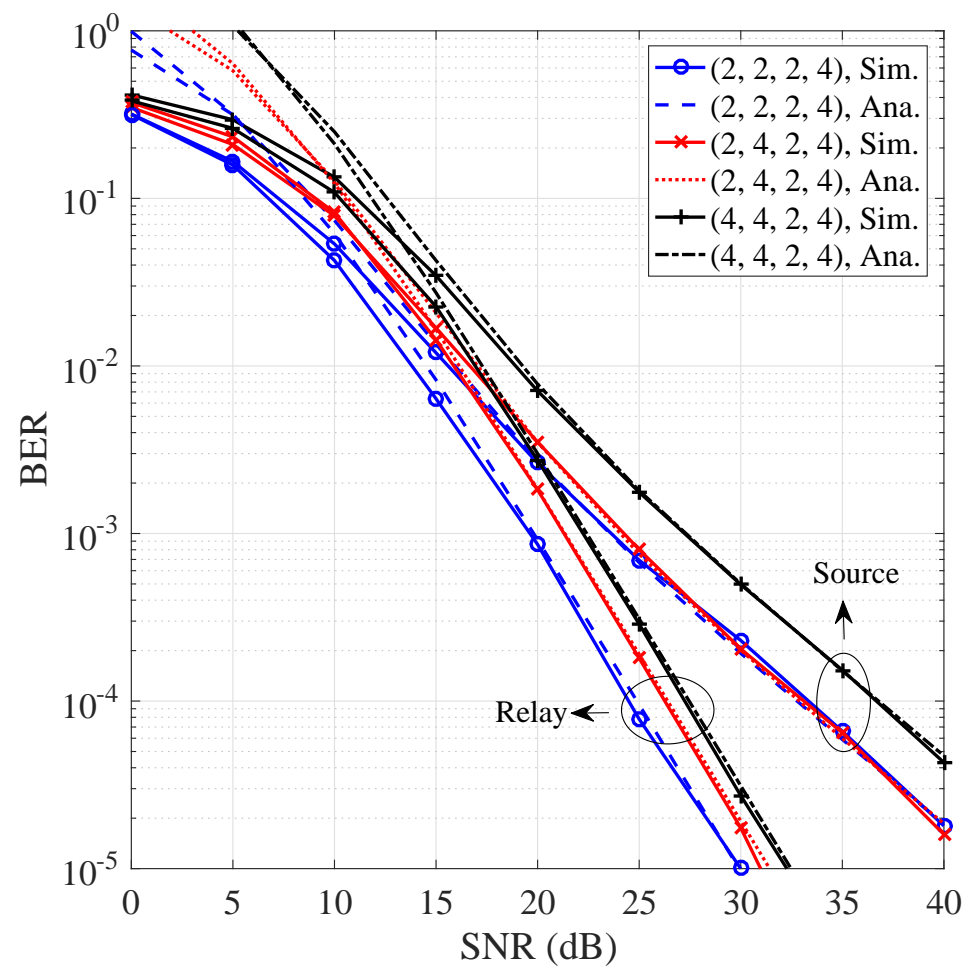

Fig. 3. Performance comparison among "RPI $(2,2,2,4)$ ", "RPI $(2,4,2,4)$ ", and "RPI $(4,4,2,4)$ " with DF relaying, where $d_{S R}=0.5$ and $d_{R D}=1$.

same data rate, SMRN-RPI outperforms SMRN-UFCP for both the source and relay, achieving SNR gains of $1 \sim 2 \mathrm{~dB}$. For both SMRN-RPI and SMRN-UFCP, the relay performs much better than the source for both values of $N_{r D}$. Since the source and relay achieve diversity orders of unity and $N_{r D}$, respectively, increasing $N_{r D}$ from 1 to 2 enhances the performance of the relay significantly, while only a slight SNR gain less than $1 \mathrm{~dB}$ is achieved by the source. This is because the overall BER performance of the source is constrained by the performance of the two hops. Increasing $N_{r D}$ has no effect on the performance of the first hop.

Fig. 3 illustrates the comparison results among "RPI $(2,2,2,4)$ ", "RPI $(2,4,2,4)$ ", and "RPI $(4,4,2,4)$ " with DF relaying, where $d_{S R}=0.5$ and $d_{R D}=1$. The analytical ABEP upper bounds are also presented. As shown in Fig. 3, all derived upper bounds agree with the simulated counterparts very well, especially at medium-to-high SNR. The performance of both the source and relay become worse with increasing $N_{t S}$ and $N_{t R}$. This phenomenon can be explained by 


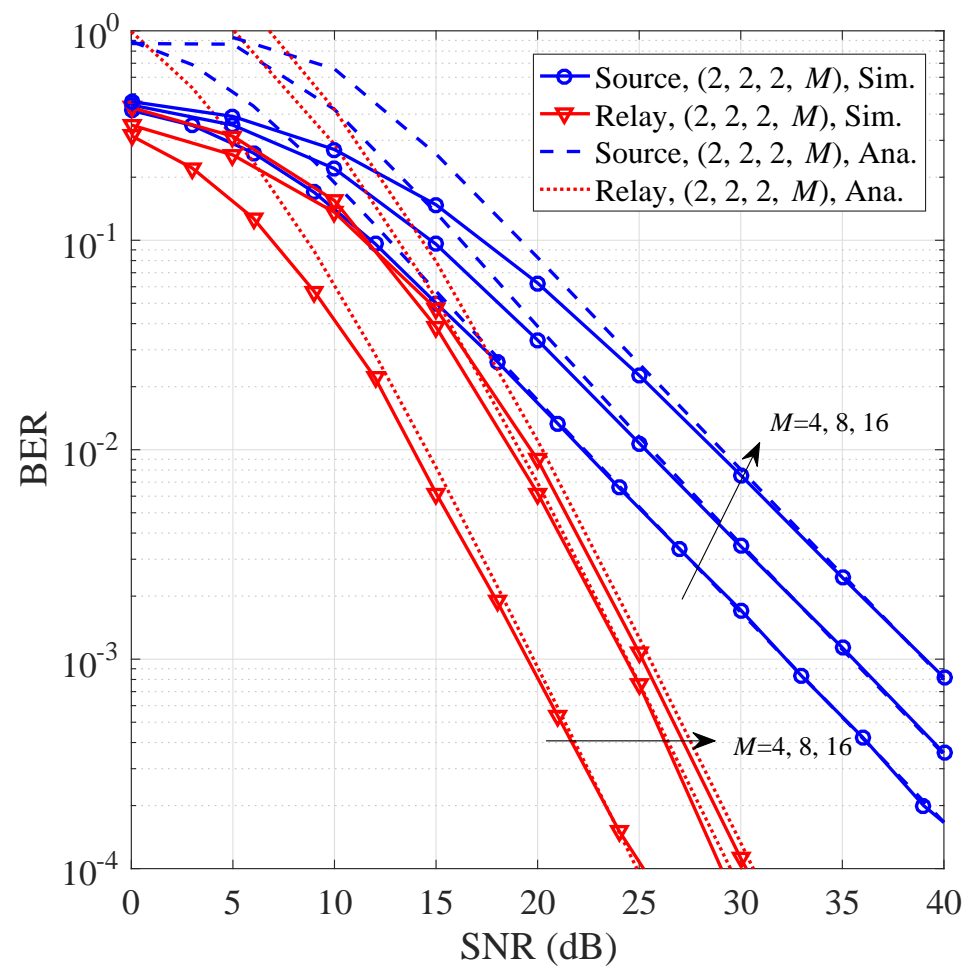

Fig. 4. Performance of "RPI $(2,2,2, M)$ " with DF relaying, where $M=4,8,16$, and $d_{S R}=d_{R D}=1$.

the fact that a larger number of available transmit antennas results in a higher error probability of detecting the active antenna indices, which is also verified by (20), (25), (30), and (40). In particular, the sources in "RPI $(2,2,2,4)$ " and "RPI $(2,4,2,4)$ " almost achieve the same asymptotic performance, which is explained in Remark 3. Besides, by comparing the curves of "RPI $(2,2,2,4)$ " in Figs. 2 and 3, we observe that the source in the case of $d_{S R}=0.5$ and $d_{R D}=1$ performs much better than that of $d_{S R}=1$ and $d_{R D}=0.5$. This is because in "RPI $(2,2,2,4)$ ", the performance of the source is highly limited by the performance of the first hop due to the single-antenna reception at the relay.

Fig. 4 gives the comparison results of "RPI $(2,2,2, M)$ " with DF relaying, where $M=4,8,16$, and $d_{S R}=d_{R D}=1$. As seen from Fig. 4, the analytical ABEP curves predict the simulation results accurately for all $M$. The source and relay achieve diversity orders of unity and two for all $M$, respectively. For both the source and relay, a larger value of $M$ results in worse performance. More specifically, at a BER value of $10^{-3}$, performance losses of about $5 \mathrm{~dB}$ and 


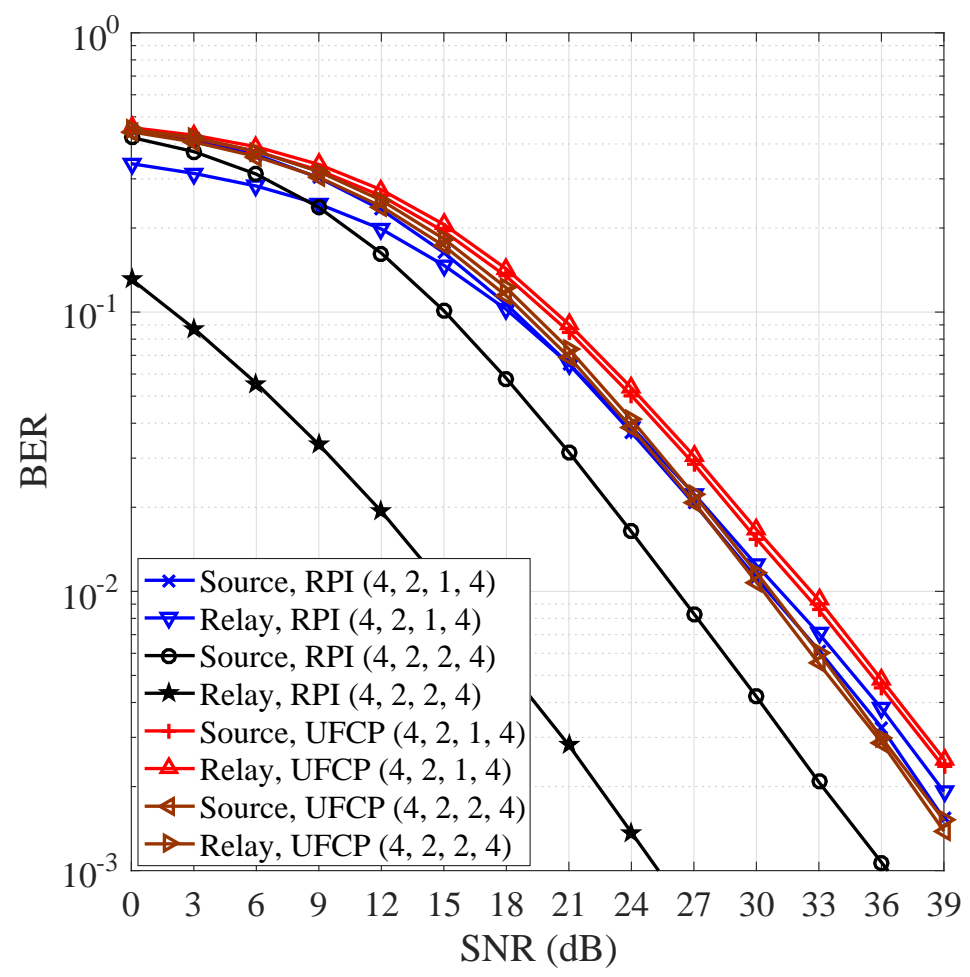

Fig. 5. Performance comparison among "RPI/UFCP $(4,2,1,4)$ " and "RPI/UFCP $(4,2,2,4)$ " with AF relaying, where the optimal ML detectors are used for all schemes, $d_{S R}=1$, and $d_{R D}=0.5$.

$1 \mathrm{~dB}$ are observed for the relay, increasing $M$ from 4 to 8 and from 8 to 16 , respectively. By contrast, both the performance losses for the source are about $3.5 \mathrm{~dB}$.

\section{B. AF Relaying}

In this subsection, we perform simulations to examine the BER performance of SMRN-RPI with AF relaying assuming the optimal/simplified ML detection. Fig. 5 presents the comparison results among "RPI/UFCP $(4,2,1,4)$ " and "RPI/UFCP $(4,2,2,4)$ " with AF relaying, where the optimal ML detectors are used for all schemes, where $d_{S R}=1$ and $d_{R D}=0.5$. The adopted UFCP for SMRN-UFCP is the same as that in Fig. 2. As seen from Fig. 5, with the same data rate, SMRN-RPI performs better than SMRN-UFCP for both $N_{r D}=1$ and $N_{r D}=2$. Although increasing $N_{r D}$ yields superior performance for both SMRN-RPI and SMRN-UFCP, the improvements are more prominent for SMRN-RPI. For $N_{r D}=1$, both the source and relay of SMRN-RPI achieve an SNR gain of about $1.5 \mathrm{~dB}$ over those of SMRN-UFCP, at a BER 


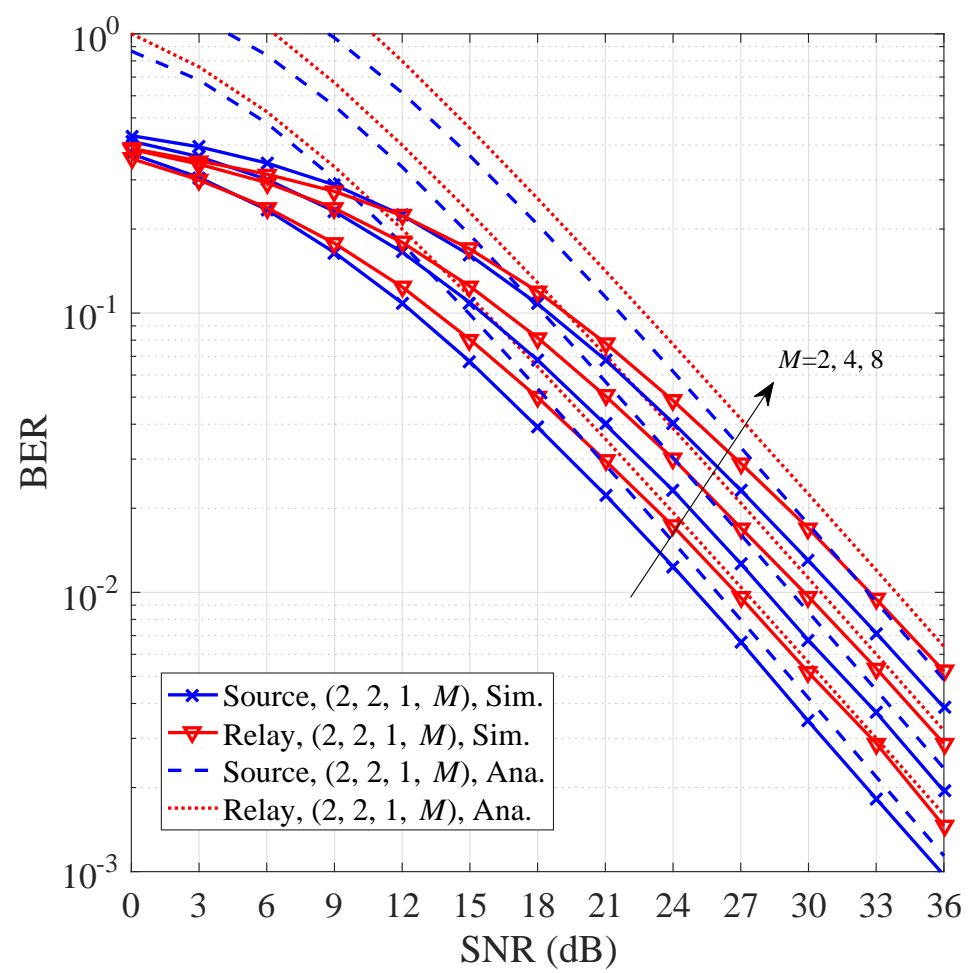

Fig. 6. Performance of "RPI $(2,2,1, M)$ " with AF relaying, where the simplified ML detectors are used for all schemes, where $M=2,4,8, d_{S R}=1$, and $d_{R D}=0.5$.

value of $10^{-2}$. By contrast, for $N_{r D}=2$, the SNR gains achieved by the source and relay of SMRN-RPI are about $4.5 \mathrm{~dB}$ and $15 \mathrm{~dB}$, respectively.

Fig. 6 depicts the performance of "RPI $(2,2,1, M)$ " with AF relaying, where $M=2,4,8$, $d_{S R}=1, d_{R D}=0.5$, and the simplified ML detectors are used for all considered schemes. Analytical upper bounds on the ABEPs of the source and relay are also presented. It can be seen from Fig. 6 that the upper bounds become tight as SNR increases, which can be accounted for by the union bounding technique. As expected, both the source and relay perform worse with increasing $M$. Specifically, for both the source and relay at a BER value of $10^{-2}$, a performance loss of about $3 \mathrm{~dB}$ is observed, increasing $M$ from 2 to 4 and from 4 to 8 . Moreover, by comparing Figs. 2 and 6, we observe that SMRN-RPI with DF relaying performs much better than that with AF relaying given the same system parameters, which is also the case in conventional relay networks. 


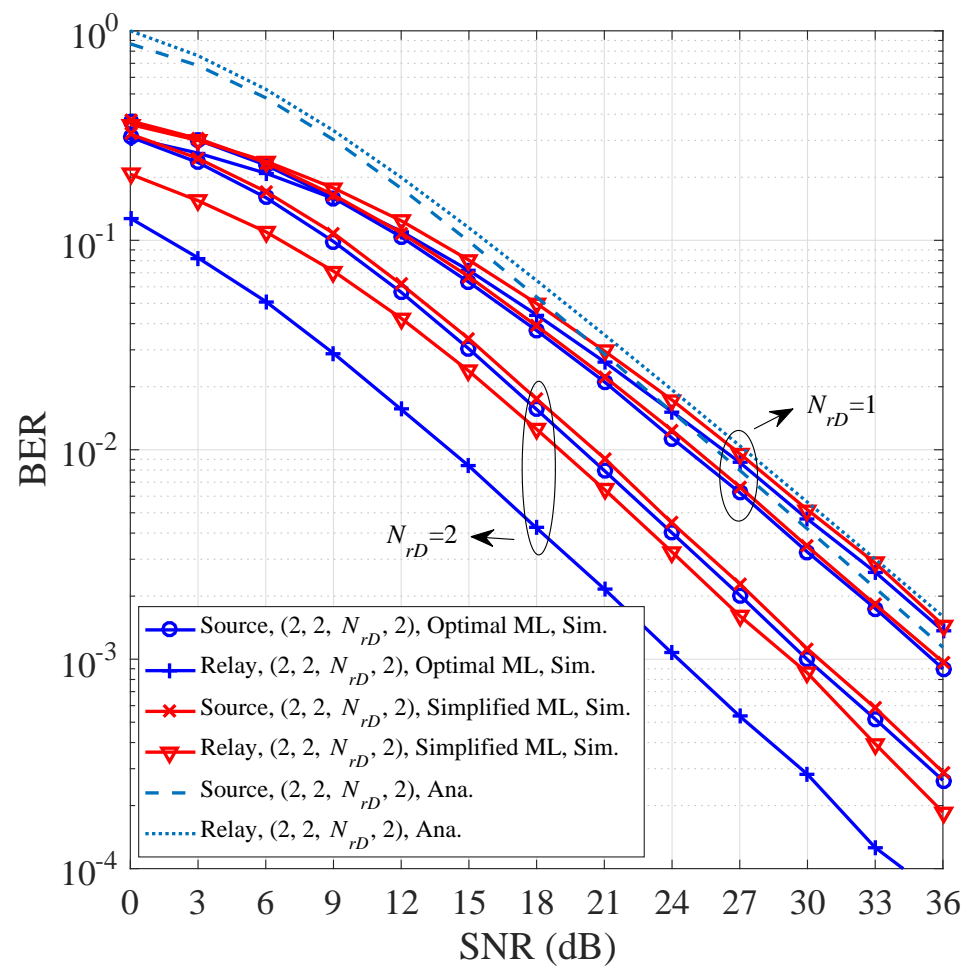

Fig. 7. Performance comparison between the optimal and simplified ML detectors of "RPI $\left(2,2, N_{r D}, 2\right)$ " with AF relaying, where $N_{r D}=1,2, d_{S R}=1$, and $d_{R D}=0.5$.

Fig. 7 depicts the performance comparisons between the optimal and simplified ML detectors of "RPI $\left(2,2, N_{r D}, 2\right)$ " with AF relaying, where $N_{r D}=1,2, d_{S R}=1$, and $d_{R D}=0.5$. The analytical ABEP upper bounds for $N_{r D}=1$ are also provided. The upper bounds accurately match with their simulation counterparts at high SNR. It can be seen from Fig. 7 that the source and relay with the optimal/simplified ML detector achieve the same diversity order of unity for both $N_{r D}=1$ and $N_{r D}=2$. Increasing $N_{r D}$ cannot improve the diversity orders of both the source and the relay. In particular, when $N_{r D}=1$, the simplified ML detector almost exhibits the same performance as the optimal ML detector for both the source and relay, and the performance loss is less than $1 \mathrm{~dB}$. By contrast, when $N_{r D}=2$, the simplified ML detector achieves near-optimal performance for the source, while the simplified ML detector performs worse than the optimal one for the relay and a performance loss of about $5 \mathrm{~dB}$ is observed. Leveraging the resulting much simplified computational complexity, the proposed simplified ML 


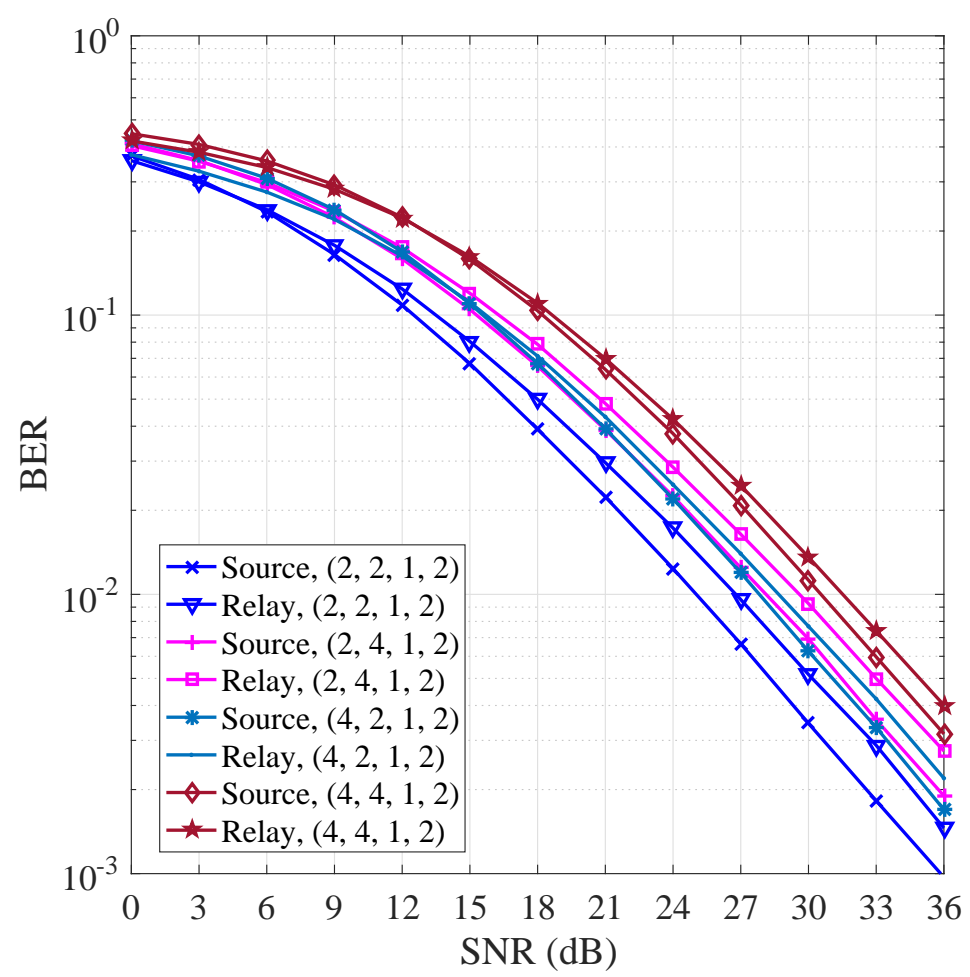

Fig. 8. Performance comparison among "RPI $(2,2,1,2)$ ", "RPI $(2,4,1,2)$ ", "RPI $(4,2,1,2)$ ", and "RPI $(4,4,1,2)$ " with AF relaying, where the simplified ML detectors are used for all schemes, $d_{S R}=1$, and $d_{R D}=0.5$.

detector is encouraging.

In Fig. 8, we make comparisons among "RPI $(2,2,1,2)$ ", "RPI $(2,4,1,2)$ ”, "RPI $(4,2,1,2)$ ", and "RPI $(4,4,1,2)$ " with AF relaying, where the simplified ML detectors are used for all schemes, $d_{S R}=1$, and $d_{R D}=0.5$. To see the effects of the numbers of transmit antennas at the source and relay clearly, the theoretical ABEP upper bounds are not included. As shown in Fig. 8, for all considered schemes, the sources achieve SNR gains of $1 \sim 2 \mathrm{~dB}$ over the relays at a BER value of $10^{-2}$. Both the source and relay perform worse with increasing $N_{t S}$ and $N_{t R}$, which can be explained by (62), (66), and (67). Increasing $N_{t S}$ or $N_{t R}$ from 2 to 4 results in a performance loss of about $3 \mathrm{~dB}$ for both the source and relay.

Fig. 9 depicts the comparison results among "(w/o) RPI $(2,2,1,4)$ ” and "(w/o) RPI $(2,2,2,4)$ ” for DF relaying, and "RPI $(4,2,1,4)$ ", “w/o RPI $(4,1,1,4)$ ", "RPI $(4,2,2,4)$ ", and "w/o RPI $(4,1,2,4)$ " for AF relaying. The optimal ML detectors are used for all schemes with $d_{S R}=1$ 


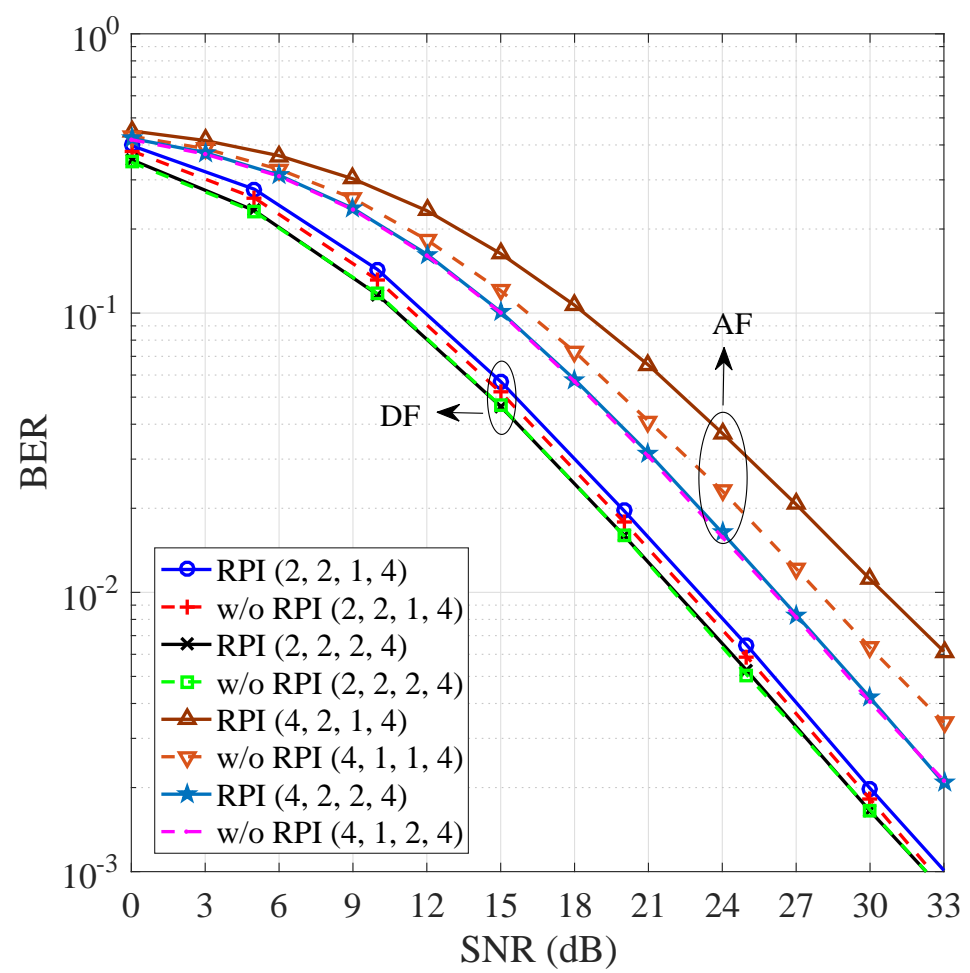

Fig. 9. Performance comparison between SMRN-RPI and the typical dual-hop SM, where the optimal ML detectors are used for both schemes, $d_{S R}=1$, and $d_{R D}=0.5$.

and $d_{R D}=0.5$. As seen from Fig. 9, when $N_{r D}=1$, there exists a performance gap between SMRN-RPI and the typical dual-hop SM, especially for AF relaying. Fortunately, when $N_{r D}=2$, the impact of added relay information is negligible for both DF and AF relaying.

In Fig. 10, we present the BER curves of SMRN-RPI versus power allocation factor $\beta$, where $P_{S}=\beta P_{T}, P_{R}=(1-\beta) P_{T}, N_{t S}=N_{t R}=2, M=4, d_{S R}=1, d_{R D}=1$, and $P_{T} / N_{0}=20$ dB. $P_{T}$ is the total power of the system. The optimal ML detectors are used for all schemes. As shown in Fig. 10, for DF relaying, as expected, the BER performance of the relay becomes worse as $\beta$ increases for both $N_{r D}=1$ and 2 . The optimal value of $\beta$ for the source increases from 0.45 to 0.7 as $N_{r D}$ increases. This is because the bottleneck in the performance of the source tends to be the first hop with increasing $N_{r D}$. On the other hand, for AF relaying with $N_{r D}=1$, coincidentally, both the optimal values of $\beta$ for the source and relay are equal to 0.4 . When $N_{r D}=2$, the optimal values of $\beta$ are changed to 0.5 and 0.15 for the source and relay, 


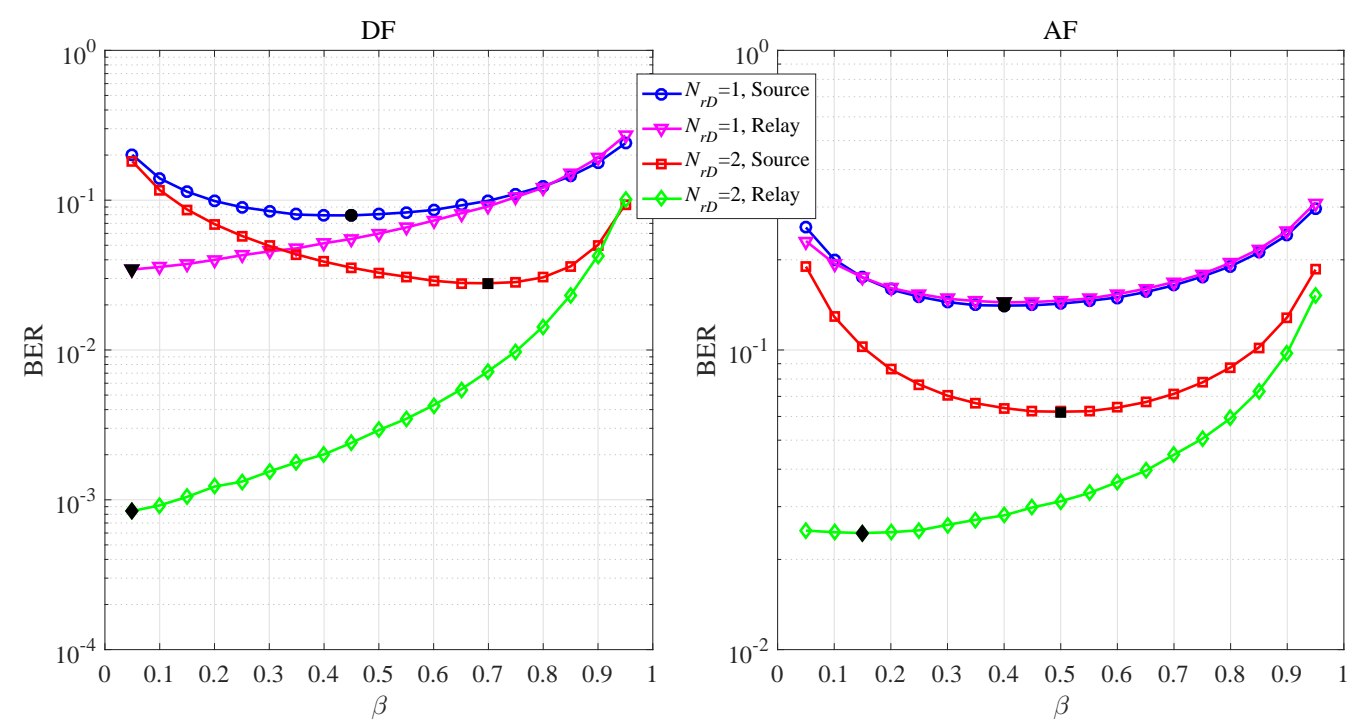

Fig. 10. Performance of SMRN-RPI versus power allocation factor $\beta$, where $P_{S}=\beta P_{T}, P_{R}=(1-\beta) P_{T}, N_{t S}=N_{t R}=$ $2, M=4, d_{S R}=1, d_{R D}=1$, and $P_{T} / N_{0}=20 \mathrm{~dB} . P_{T}$ is the total power of the system.

respectively.

\section{CONCLUSIONS AND REMARKS}

In this paper, we have proposed a novel dual-hop SM relay network, in which SM is invoked at the source, and the relay concurrently transmits its own private information in addition to forwarding the received SM signals to the destination. Both DF and AF relaying protocols have been developed for the relay. For both DF and AF relaying, tight upper bounds on the ABEPs of the source and relay have been derived in closed form over Rayleigh flat fading channels. The performance analysis has been verified by computer simulations. We conclude that the proposed SMRN-RPI provides a simple yet effective solution to the implementation of SM relay networks in which the relay has its own information to be transmitted. We remark that the end-to-end performance of the source in SMRN-RPI is highly limited by the signal quality of the first hop. It can be predicted that the performance of the source can be greatly improved by using multi-antenna reception at the relay. Maximal ratio combining and selection combining can be employed for DF and AF relaying, respectively. However, this is an issue of interest for future study. 


\section{APPENDIX A}

\section{ProOF OF PROPOSITION 1}

Since $\mathbf{h}$ and $\mathbf{g}$ are independent of each other, we have

$$
\mathcal{M}_{\lambda_{i}}(t)=E\left\{\exp \left(\lambda_{i} t\right)\right\}=E_{\mathbf{g}}\left\{E_{\mathbf{h} \mid \mathbf{g}}\left\{\exp \left(\lambda_{i} t\right)\right\}\right\}, \quad i=1,2,3,4 .
$$

Note that $\left|h_{l}\right|^{2}$ in $\lambda_{1}$ and $\left|h_{l} s-h_{\hat{l}} \hat{s}\right|^{2}$ in $\lambda_{2}$ are exponential random variables with means equal to $\Omega_{S R}$ and $2 \Omega_{S R}$, respectively. Hence, using the MGF approach, we have

$$
E_{\mathbf{h} \mid \mathbf{g}}\left\{\exp \left(\lambda_{i} t\right)\right\}=\frac{C+\left|g_{m}\right|^{2}}{C+\omega_{i}\left|g_{m}\right|^{2}}, \quad i=1,2
$$

where $\omega_{1}(t)=1-|s-\hat{s}|^{2} \Omega_{S R} t$ and $\omega_{2}(t)=1-2 \Omega_{S R} t$. Finally, by averaging (73) over $\left|g_{m}\right|^{2}$, whose PDF is given by $f_{\left|g_{m}\right|^{2}}(v)=\exp \left(-v / \Omega_{R D}\right) / \Omega_{R D}$, and using [33, Eqs. (3.352-4, 3.353-5)], we are led to (51).

\section{APPENDIX B}

\section{PROOF OF PROPOSITION 2}

For $P_{S} \Omega_{S R} \ll P_{R} \Omega_{R D}$, we have $C \ll\left|g_{m}\right|^{2}$, such that $\lambda_{3}$ can be approximated as

$$
\lambda_{3} \approx \frac{\left|h_{l}\right|^{2}\left|g_{m} s-g_{\hat{m}} \hat{s}\right|^{2}}{\left|g_{m}\right|^{2}} .
$$

It can be shown that in (74), $\left|g_{m} s-g_{\hat{m}} \hat{s}\right|^{2}$ and $\left|g_{m}\right|^{2}$ are two correlated exponentially distributed random variables with correlation coefficient $1 / 2$, and $\left|h_{l}\right|^{2}$ is an independent exponential random variable. The CDF of $\lambda_{3}$ can be derived as [28]

$F_{\lambda_{3}}(v)=\frac{\Omega_{S R}}{2}-\frac{\pi v}{8}\left[\mathbf{H}_{1}\left(\frac{v}{2 \Omega_{S R}}\right)-Y_{1}\left(\frac{v}{2 \Omega_{S R}}\right)\right]+\frac{v}{4}+\frac{\pi v}{8}\left[\mathbf{H}_{0}\left(\frac{v}{2 \Omega_{S R}}\right)-Y_{0}\left(\frac{v}{2 \Omega_{S R}}\right)\right]$.

Based on $F_{\lambda_{3}}(v)$, the MGF of $\lambda_{3}$ can be obtained from (75) as

$$
\begin{aligned}
\mathcal{M}_{\lambda_{3}}(-t)=t \cdot \mathcal{L}\left\{F_{\lambda_{3}}(v)\right\}= & \frac{\Omega_{S R}}{2}-\frac{1}{8 \Omega_{S R}^{2} t^{3}}{ }_{3} F_{2}\left(1,2,5 / 2 ; 3 / 2,5 / 2 ;-\frac{1}{4 \Omega_{S R}^{2} t^{2}}\right) \\
& -t\left(\frac{1}{2 \Omega_{S R}^{2}}+2 t^{2}\right)^{-1} Q_{1}^{-1}\left(t\left(\frac{1}{4 \Omega_{S R}^{2}}+t^{2}\right)^{-1 / 2}\right) \\
& +\frac{1}{4 t}+\frac{1}{4 \Omega_{S R} t^{2}}{ }_{3} F_{2}\left(1,3 / 2,2 ; 3 / 2,3 / 2 ;-\frac{1}{4 \Omega_{S R}^{2} t^{2}}\right) \\
& +t\left(\frac{1}{\Omega_{S R}^{2}}+4 t^{2}\right)^{-1} Q_{1}^{0}\left(t\left(\frac{1}{4 \Omega_{S R}^{2}}+t^{2}\right)^{-1 / 2}\right),
\end{aligned}
$$


where the integrals [33, Eqs. (6.823-1, 6.621-2)] are applied. From (76), we can obtain (52).

For $P_{S} \Omega_{S R} \gg P_{R} \Omega_{R D}$, we have

$$
\lambda_{3} \approx \frac{\left|h_{l}\right|^{2}\left|g_{m} s-g_{\hat{m}} \hat{s}\right|^{2}}{C} .
$$

Recalling that $\left|h_{l}\right|^{2}$ and $\left|g_{m} s-g_{\hat{m}} \hat{s}\right|^{2}$ are exponential random variables with means equal to $\Omega_{S R}$ and $2 \Omega_{R D}$, respectively, with (72), the MGF of $\lambda_{3}$ in (77) can be derived as

$$
\begin{aligned}
\mathcal{M}_{\lambda_{3}}(t) & =E_{\mathrm{g}}\left\{\frac{C}{C-\left|g_{m} s-g_{\hat{m}} \hat{s}\right|^{2} \Omega_{S R} t}\right\} \\
& =-\frac{C}{2 \Omega_{S R} \Omega_{R D} t} \exp \left(-\frac{C}{2 \Omega_{S R} \Omega_{R D} t}\right) \mathrm{E}_{1}\left(-\frac{C}{2 \Omega_{S R} \Omega_{R D} t}\right),
\end{aligned}
$$

which completes the proof.

\section{APPENDIX C}

\section{PROOF OF PROPOSITION 3}

Given $\mathbf{g},\left|h_{l} s g_{m}-h_{\hat{l}} \hat{s} g_{\hat{m}}\right|^{2}$ in $\lambda_{4}$ is an exponentially distributed random variable with mean equal to $\left(\left|g_{m}\right|^{2}+\left|g_{\hat{m}}\right|^{2}\right) \Omega_{S R}$. Therefore, using the MGF approach yields

$$
E_{\mathbf{h} \mid \mathbf{g}}\left\{\exp \left(\lambda_{4} t\right)\right\}=\frac{C+\left|g_{m}\right|^{2}}{C+\left|g_{m}\right|^{2}-\Omega_{S R}\left|g_{m}\right|^{2} t-\Omega_{S R}\left|g_{\hat{m}}\right|^{2} t} .
$$

According to (72), the MGF of $\lambda_{4}$ can be obtained by averaging (79) over $\left|g_{m}\right|^{2}$ and $\left|g_{\hat{m}}\right|^{2}$, both of which are exponentially distributed with mean equal to $\Omega_{R D}$. Hence, we have

$$
\begin{aligned}
& E_{\left.\left|g_{\hat{m}}\right|^{2}|| g_{m}\right|^{2}}\left\{\frac{C+\left|g_{m}\right|^{2}}{C+\left|g_{m}\right|^{2}-\Omega_{S R}\left|g_{m}\right|^{2} t-\Omega_{S R}\left|g_{\hat{m}}\right|^{2} t}\right\} \\
& =-\frac{C+\left|g_{m}\right|^{2}}{\Omega_{S R} \Omega_{R D} t} \exp \left(\frac{\Omega_{S R}\left|g_{m}\right|^{2} t-\left|g_{m}\right|^{2}-C}{\Omega_{S R} \Omega_{R D} t}\right) \mathrm{E}_{1}\left(\frac{\Omega_{S R}\left|g_{m}\right|^{2} t-\left|g_{m}\right|^{2}-C}{\Omega_{S R} \Omega_{R D} t}\right) .
\end{aligned}
$$

Using the Lemma and averaging (80) over $\left|g_{m}\right|^{2}$ yields (59), completing the proof.

\section{REFERENCES}

[1] M. Wen, B. Zheng, K. J. Kim, M. Di Renzo, T. A. Tsiftsis, K.-C. Chen, and N. Al-Dhahir, "A survey on spatial modulation in emerging wireless systems: Research progresses and applications," IEEE J. Sel. Areas Commun., vol. 37, no. 9, pp. 19491972, Sep. 2019.

[2] M. Di Renzo, H. Haas, A. Ghrayeb, S. Sugiura, and L. Hanzo, "Spatial modulation for generalized MIMO: Challenges, opportunities and implementation,” Proc. IEEE, vol. 102, no. 1, pp. 56-103, Jan. 2014.

[3] R. Y. Mesleh, H. Haas, S. Sinanovic, C. W. Ahn, and S. Yun, "Spatial modulation," IEEE Trans. Veh. Technol., vol. 57, no. 4, pp. 2228-2241, Jul. 2008. 
[4] M. Wen, X. Cheng, and L. Yang, Index Modulation for 5G Wireless Communications. Springer International Publishing AG, Cham, Switzerland, 2017.

[5] X. Cheng, M. Zhang, M. Wen, and L. Yang, "Index modulation for 5G: Striving to do more with less," IEEE Wireless Commun. Mag., vol. 25, no. 2, pp. 126-132, Apr. 2018.

[6] E. Basar, M. Wen, R. Mesleh, M. Di Renzo, Y. Xiao, and H. Haas, "Index modulation techniques for next-generation wireless networks,” IEEE Access, vol. 5, pp. 16693-16746, Sep. 2017.

[7] M. Di Renzo and H. Haas, "Bit error probability of SM-MIMO over generalized fading channels," IEEE Trans. Veh. Technol., vol. 61, no. 3, pp. 1124-1144, Mar. 2012.

[8] A. Younis, N. Serafimovski, R. Mesleh, and H. Haas, “Generalised spatial modulation,” in Proc. Asilomar Conf. Signals, Syst., Comput., Pacific Grove, CA, USA, Nov. 2010, pp. 1498-1502.

[9] J. Wang, S. Jia, and J. Song, "Generalised spatial modulation system with multiple active transmit antennas and low complexity detection scheme,” IEEE Trans. Wireless Commun., vol. 11, no. 4, pp. 1605-1615, Apr. 2012.

[10] R. Zhang, L.-L. Yang, and L. Hanzo, "Generalised pre-coding aided spatial modulation," IEEE Trans. Wireless Commun., vol. 12, no. 11, pp. 5434-5443, Nov. 2013.

[11] Y. Bian, X. Cheng, M. Wen, L. Yang, H. Vincent Poor, and B. Jiao, "Differential spatial modulation," IEEE Trans. Veh. Technol., vol. 64, no. 7, pp. 3262-3268, Jul. 2015.

[12] S. Sugiura, S. Chen, and L. Hanzo, "Coherent and differential space-time shift keying: A dispersion matrix approach," IEEE Trans. Commun., vol. 58, no. 11, pp. 3219-3230, Nov. 2010.

[13] J. Jeganathan, A. Ghrayeb, L. Szczecinski, and A. Ceron, "Space shift keying modulation for MIMO channels," IEEE Trans. Wireless Commun., vol. 8, no. 7, pp. 3692-3703, Jul. 2009.

[14] S. Narayanan, M. Di Renzo, M. J. Chaudhry, F. Graziosi, and H. Haas, "On the achievable performance-complexity tradeoffs of relay-aided space shift keying," IEEE Trans. Signal and Information Processing over Networks, vol. 1, no. 2, pp. 129-144, Jun. 2015.

[15] R. Mesleh, S. S. Ikki, and H. M. Aggoune, "Quadrature spatial modulation,” IEEE Trans. Veh. Technol., vol. 64, no. 6, pp. 2738-2742, Jun. 2015.

[16] S. Dang, J. P. Coon, and G. Chen, "Adaptive OFDM with index modulation for two-hop relay-assisted networks," IEEE Trans. Wireless Commun., vol. 17, no. 3, pp. 1923-1936, Mar. 2018.

[17] S. Dang, J. Li, M. Wen, S. Mumtaz, and Z. Zhang, "OFDM-IM based dual-hop system using fixed-gain amplify-and-forward relay with pre-processing capability," IEEE Trans. Wireless Commun., vol. 18, no. 4, pp. 2259-2270, Apr. 2019.

[18] N. Serafimovski, S. Sinanovic, M. Di Renzo, and H. Haas, "Dual-hop spatial modulation (Dh-SM)," in Proc. IEEE Veh. Technol. Conf., Yokohama, Japan, May 2011, pp. 1-5.

[19] P. Yang, B. Zhang, Y. Xiao, B. Dong, S. Li, M. El-Hajjar, and L. Hanzo, "Detect-and-forward relaying aided cooperative spatial modulation for wireless networks," IEEE Trans. Commun., vol. 61, no. 11, pp. 4500-4511, Nov. 2013.

[20] R. Mesleh and S. S. Ikki, "Performance analysis of spatial modulation with multiple decode and forward relays," IEEE Wireless Commun. Lett., vol. 2, no. 4, pp. 423-426, Aug. 2013.

[21] A. Stavridis, D. Basnayaka, S. Sinanovic, M. Di Renzo, and H. Haas, "A virtual MIMO dual-hop architecture based on hybrid spatial modulation," IEEE Trans. Commun., vol. 62, no. 9, pp. 3161-3179, Sep. 2014.

[22] Q. Li, M. Wen, E. Basar, H. V. Poor, and F. Chen, "Spatial modulation-aided cooperative NOMA: Performance analysis and comparative study,“ IEEE J. Sel. Topics Signal Process., vol. 13, no. 3, pp. 715-728, Jun. 2019. 
[23] X. Yu, Q. Pan, S.-H. Leung, and C. Wang, "Power allocation and performance analysis of cooperative spatial modulation in wireless relay networks," IEEE Access, vol. 6, pp. 12145-12155, Mar. 2018.

[24] A. Afana, R. Mesleh, S. Ikki, and I. E. Atawi, "Performance of quadrature spatial modulation in amplify-and-forward cooperative relaying," IEEE Commun. Lett., vol. 20, no. 2, pp. 240-243, Feb. 2016.

[25] S. Narayanan, H. Ahmadi, and M. F. Flanagan, "On the performance of spatial modulation MIMO for full-duplex relay networks," IEEE Trans. Wireless Commun., vol. 16, no. 6, pp. 3727-3746, Jun. 2017.

[26] P. Raviteja, Y. Hong, and E. Viterbo, "Spatial modulation in full-duplex relaying," IEEE Commun. Lett., vol. 20, no. 10, pp. 2111-2114, Oct. 2016.

[27] A. Koc, I. Altunbas, and E. Basar, "Two-way full-duplex spatial modulation systems with wireless powered AF relaying," IEEE Wireless Commun. Lett., vol. 7, no. 3, pp. 444-447, Jun. 2018.

[28] A. Alizadeh, H. R. Bahrami, and M. Maleki, "Performance analysis of spatial modulation in overlay cognitive radio communications," IEEE Trans. Commun., vol. 64, no. 8, pp. 3220-3232, Aug. 2016.

[29] S. Ustunbas, E. Basar, and U. Aygolu, "Cooperative spectrum sharing protocol using spatial modulation," IET Commun., vol. 11, no. 11, pp. 1759-1767, Sep. 2017.

[30] S. Narayanan, M. Di Renzo, F. Graziosi, and H. Haas, "Distributed spatial modulation: A cooperative diversity protocol for half-duplex relay-aided wireless networks," IEEE Trans. Veh. Technol., vol. 65, no. 5, pp. 2947-2964, May 2016.

[31] W. Yan, X. Yuan, and X. Kuai, "Passive beamforming and information transfer via large intelligent surface," IEEE Wireless Commun. Lett., to be published, 2020.

[32] D. G.-Barquero, Next Generation Mobile Broadcasting. Boca Raton, FL, USA: CRC Press, 2013.

[33] I. S. Gradshteyn and I. M. Ryzhik, Table of Integrals, Series, and Products, 7th ed., New York, NY, USA: Academic Press, 2007.

[34] M. Maleki, H. R. Bahrami, A. Alizadeh, and N. H. Tran, "On the performance of spatial modulation: Optimal constellation breakdown,” IEEE Trans. Commun., vol. 62, no. 1, pp. 144-157, Jan. 2014.

[35] Q. Li, M. Wen, E. Basar, and F. Chen, "Index modulated OFDM spread spectrum," IEEE Trans. Wireless Commun., vol. 17, no. 4, pp. 2360-2374, Apr. 2018.

[36] M. K. Simon, Probability Distributions Involving Gaussian Random Variables: A Handbook for Engineers and Scientists. Berlin, Germany: Springer, 2006.

[37] M.-S. Alouini and A. J. Goldsmith, "A unified approach for calculating error rates of linearly modulated signals over generalized fading channels," IEEE Trans. Commun., vol. 47, no. 9, pp. 1324-1334, Sep. 1999.

[38] M. K. Simon and M.-S. Alouini, Digital Communication Over Fading Channels, 2nd ed. New York, NY, USA: Wiley, 2005 .

[39] A. A. Alkheir and M. Ibnkahla, "An accurate approximation of the exponential integral function using a sum of exponentials," IEEE Commun. Lett., vol. 17, no. 7, pp. 1364-1367, Jul. 2013.

[40] M. O. Hasna and M.-S. Alouini, "A performance study of dual-hop transmissions with fixed gain relays," IEEE Trans. Wireless Commun., vol. 3, no. 6, pp. 1963-1968, Nov. 2004.

[41] D. Xia, J.-K. Zhang, and S. Dumitrescu, "Energy-efficient full diversity collaborative unitary space-time block code designs via unique factorization of signals," IEEE Trans. Inf. Theory, vol. 59, no. 3, pp. 1678-1703, Mar. 2013. 